\title{
Role of plates and temperature-dependent viscosity in phase change dynamics
}

\author{
Shijie Zhong and Michael Gurnis 1 \\ Department of Geological Sciences, University of Michigan, Ann Arbor
}

\begin{abstract}
The effects of plates and slabs on phase change dynamics have been investigated with convection models. Two complementary methods to simulate plates are used: material property and imposed surface velocity methods with temperature-dependent viscosity. For a wide range of model parameters, plates and slabs exert a significant control on phase change dynamics. As plate length (and hence plate age and convection cell aspect ratio) increases, both the propensity for slab penetration and the mass flux actoss an endothermic phase change increase. When cold downwellings are stiffened with a temperature-dependent rheology, slab penetration is enhanced, but total mass flux changes little. Plates organize large-scale flow and thermal structure and thereby affect phase change dynamics. As plates become larger, the resulting largerscale structures are influcnced less by endothermic phase changes, thus reducing the degree of layering. A model showing completely layered convection for a plate of unit length becomes unlayered when the plate is 3 or 5 times longer. For a given Clapeyron slope, the proportion of time for slab penetration increases from zero for cases with small plates to more than 0.5 for cases with large plates. The degree of layering, plate velocity, and mass flux are controlled by large-scale structures, while slab penetration may be more related to small-scale features. Therefore, whether or not subducted slabs penetrate the phase change may not necessarily indicate that convection is entirely layered or entirely unlayered. The episodicity of convection induced by an endothermic phase change strongly depends on plate length, rheology, and Clapeyron slope. A large plate and a stiff slab both weaken the episodicity of convection. Only for a certain range of Clapeyron slopes can the phase change induce a strong episodic thermal convection.
\end{abstract}

\section{Introduction}

Experimental petrology and the seismic velocity structure through the upper mantle suggest that the discontinuities at $410 \mathrm{~km}$ and $670 \mathrm{~km}$ probably result from solid-solid phase transitions from olivine to spinel and from spinel to a postspinel phase, respectively. The discontinuity at $670 \mathrm{~km}$ is of particular importance, since it would tend to retard mantle convection if it were endothermic [Schubert et al., 1975]. Earlier studies have shown that the nature of an endothermic phase change at $670 \mathrm{~km}$ depth may determine if mantle convection is layered or if subducting slabs can penetrate into the lower mantle. With a two-dimensional, unit aspect ratio model, convection is layered and cold downwelling limbs are inhibited from penetrating into the lower layer, if the Clapeyron slope of the endothermic phase change is larger than 4-8 $\mathrm{MPa} \mathrm{K}^{-1}$ [Christensen and Yuen, 1984; 1985]. In models with a long box, multiple phase transitions, and depth-dependent thermal expansivity, the tendency for layered convection increases [Zhao et al., 1992]. Recently, attention has focused on the time dependence induced by an endothermic phase change. In a spherically axisymmetric model, layered convection is intermittent, and strong downwellings

\footnotetext{
${ }^{1}$ Now at Seismological Laboratory, California Institute of Technology, Pasadena.
}

Copyright 1994 by the American Geophysical Union.

Paper number 94JB00545.

0148-0227/94/94JB-00545\$05.00 occasionally penetrate an endothermic phase boundary at 670 $\mathrm{km}$ depth [Machetel and Weber, 1991]. Intermittent layered convection has been found in various other models [Christensen and Yuen, 1985; Weinstein, 1993; Tackley et al., 1993; Honda et al., 1993]. In a three-dimensional spherical shell model, Tackley et al. [1993] reported that cold downwellings are temporarily stopped by the $670-\mathrm{km}$ endothermic phase change but sink rapidly into the lower mantle.

The intermittence of layering reflects accumulation and release of negative buoyancy above the endothermic phase boundary [Machetel and Weber, 1991; Tackley et al., 1993]. For cold downwellings, the endothermic phase boundary curves downward to generate a buoyancy retarding penetration. When penetration is retarded, cold material accumulates above the endothermic phase boundary. Simultaneously, hot upwellings are generally suppressed under the phase boundary. When this accumulated negative buoyancy dominates over that of the phase boundary deflection, the cold material penetrates into the lower layer; simultaneously, the hot upwelling fluid will be pushed into the upper layer. The exchange of mass between upper and lower layers can occur in short bursts (often described with superlatives such as "catastrophic" overturns, "avalanche" subduction, or "superplumes"). As the negative buoyancy is released, buoyancy associated with the deflection of phase boundary dominates and layering returns.

Whether convection is layered or not depends largely on whether the dominant buoyancy near the phase boundary is the negative thermal buoyancy or the positive phase change buoyancy. The negative thermal buoyancy results from the cooling of oceanic plates; the positive buoyancy depends on 
the Clapeyron slope and density jump associated with the phase change at $670 \mathrm{~km}$ depth, which can be constrained from experimental petrology and seismic velocity structure, respectively.

As oceanic plates move away from ridges, they cool and acquire a negative buoyancy, which is evident from the relation between lithospheric age, seafloor subsidence, and heat flow [Parsons and Sclater, 1977]. Oceanic plates continue to retain negative buoyancy along their way from oceanic ridges to oceanic trenches where they subduct into the mantle. With the temperature- and stress-dependent rheology of silicate rocks, oceanic plates are sufficiently strong such that they are rigid everywhere except at plate boundaries; the stiffness of oceanic plates gives plates two fundamental properties [Davies, 1988a]: (1) the horizontal dimension of oceanic plates can be much larger than the thickness of the mantle, e.g., Pacific plate is as long as $14,000 \mathrm{~km}$; and (2) most of the negative buoyancy obtained while plates move away from oceanic ridges is retained within the plates, and the negative buoyancy will not be released until the plates subduct into the mantle. This suggests that the longer (or older) a plate is, the more negative buoyancy the plate has prior to subduction. It should be pointed out that the "flattening" of oceanic age-depth relation for seafloor older than $70 \mathrm{Ma}$ [Parsons and Sclater, 1977] does not necessarily imply that plates do not cool after $70 \mathrm{Ma}$. The "flattening" can be attributed to the effects of hotspots [Heestand and Crough, 1981; Schroeder, 1984] and other dynamic processes under oceanic plates [Davies, 1988b, c; Cazenave and Lago, 1991; Davies and Pribac, 1993]. Recently, several studies on global seismic velocity structure also indicate that oceanic plates continue to cool after $70 \mathrm{Ma}$. Woodward and Masters [1991] reported that the differential $S S-S$ and $P P-P$ travel time residuals decrease almost linearly with square root of seafloor age to about $150 \mathrm{Ma} ; S u$ and Dziewonski [1992] reported similar results for $S S$ residuals. The decrease in travel time residuals implies an increase in seismic velocities, which suggests a density increase or a temperature decrease. Zhang and Tanimoto [1991] showed that phase velocities of Love waves which primarily sample the shallow structure continuously increase with age of oceanic plates to $150 \mathrm{Ma}$.

While many numerical studies [e.g., Weinstein, 1993; Tackley et al., 1993] have used realistic mantle material properties including the Clapeyron slope and density jump of the $670-\mathrm{km}$ phase change, oceanic plates are poorly simulated in all studies with constant viscosity. Constant viscosity inherently precludes the formation of long and rigid oceanic plates. For models with a Rayleigh number larger than $10^{6}$, a top thermal boundary layer quickly breaks down into small downwelling cold limbs, and the characteristic length between these top thermal boundary layers is usually comparable to the thickness of the mantle (see typical thermal structures of Peltier and Solheim [1992] and Machetel and Weber [1991]), which is thus considerably shorter than that of the Pacific plate. For those models with unit aspect ratio [e.g., Christensen and Yuen, 1985], the length of top thermal boundary layer is naturally smaller than the thickness of the box. As pointed out above, a shorter plate would give less negative buoyancy within the plate prior to subduction and hence less chance for the subducting slab to penetrate into the lower mantle. Moreover, it has been shown that plate-scale flow and thermal structures induced by the motion of rigid plates are the predominant structures within the mantle and are essential to explaining surface observables including heat flux, seafloor subsidence, and geoid [Davies, 1988a, b, c]. Indeed, in any great circle profile of the Earth's mantle, there are no more than three subduction zones. However, in most previous isoviscous models with phase changes, thermal structure within the mantle is dominated by small-scale structure [e.g., Peltier and Solheim, 1992] which leads to great difficulties in explaining surface observables [Davies, 1988c]. Even in the three-dimensional spherical shell models of Tackley et al. [1993], although thermal structure is characterized by relatively large aspect ratio features, there are still on average more than six downwellings per great circle path. Models with large plates are essential if comparisons are made with observations, since most of our seismological understanding of the transition zone comes from the western Pacific where the oceanic lithosphere is old. Since phase changes may have less effects on large-scale thermal structure than on small-scale plume structures, the dominant large-scale flow and thermal structures may result in different phase change dynamics than do the small-scale structures from isoviscous convection models. Isoviscous models have also failed to incorporate slab strength. Subducted slabs are stiffer than ambient mantle due to their lower temperature; stiffer slabs have more capacity to retain their integrity and perhaps may have a greater ability to penetrate into the lower mantle [Christensen and Yuen, 1984]. It is clearly essential to study phase change dynamics in the presence of both long plates and stiff slabs.

Realistic plates and subducted slabs have been incorporated into two-dimensional Cartesian models with phase changes. A successful plate model must be capable of producing "platelike" surface deformation; i.e., strain is concentrated at plate boundaries and is insignificant within the plate interior. For relatively small size plates, various methods including material properties [Gumis and Hager, 1988; King and Hager, 1990], force balance [Gable et al., 1991], and power- law thin sheets [Weinstein and Olson, 1992] have been used [King et al., 1992]. For large size plates, Davies [1988a] used imposed surface velocity with temperature-dependent viscosity. In the material property method, the oceanic plates are simulated by imposing high viscosity for the top boundary layer and two weak margins on the periphery; the length of plates can be varied by changing the aspect ratio of the box. Although the material property method is an effective method to simulate plates, it cannot easily account for stiff slabs. An imposed surface velocity method with temperature-dependent viscosity can simulate both plates and stiff slabs, but it is computationally costly. In this study, both the material property method and the imposed surface velocity method with temperature-dependent viscosity are used to investigate different effects of plates and stiff slabs on phase change dynamics.

Our computations for a wide range of parameters show that plates and stiff slabs significantly affect phase change dynamics. As plate length increases, mass flux across the endothermic phase change increases, degree of layering decreases, and slab penetration occurs more frequently. Stiff slabs enhance slab penetration substantially but have little effects on global features including the total mass flux and degree of layering. Finally, large size plates can be well simulated using material property methods, even in the presence of both endothermic and exothermic phase changes. 


\section{Physical Models and Method}

\section{Equations and Model Setup}

The governing equations for mantle convection are derived from the conservation of mass, momentum and energy. Because the mantle has a very high Prandtl number, the inertial terms in the momentum equation are negligible. With the Boussinesq approximation, the fluid is effectively incompressible [McKenzie et al, 1974]. The momentum, continuity, and energy equations are, respectively,

$$
\begin{aligned}
& \sigma_{i j, j}+f_{i}=0, \\
& u_{i, i}=0, \\
& \frac{\partial T}{\partial t}+u_{i} \cdot T_{, i}=\kappa T_{, i i}+H,
\end{aligned}
$$

where $u_{i}, f_{i}$, and $\sigma_{i j}$ are the flow velocity, the body force, and the stress tensor, respectively; $T$ is the temperature; $t$ is the time; $\kappa$ is the thermal diffusivity; $H$ is the heat source; and $i$ and $j$ are spatial indices. Throughout the paper, $A,,_{i}$ represents partial derivative of function $A$ with respect to coordinate $x_{i}$; repeated spatial indices denote summation. The body force vector and the stress tensor may be expressed in terms of an equation of state and a constitutive equation as

$$
f_{i}=-\rho_{0}\left[1-\alpha\left(T-T_{0}\right)+\sum_{k} \Gamma_{k} \frac{\Delta \rho_{k}}{\rho_{0}}\right] g \delta_{i 2},
$$

and

$$
\sigma_{i j}=-P \delta_{i j}+\mu\left(u_{i, j}+u_{j, i}\right)
$$

where $\rho_{0}$ and $T_{0}$ are the reference density and temperature, respectively; $g$ is the gravitational acceleration; $\alpha$ is the coefficient of thermal expansion; $k$ is the index for phase change; $\Gamma_{k}$ and $\Delta \varphi_{k}$ are the dimensionless phase change function and density jump for the $k$ th phase change, respectively; $P$ is the pressure; and $\mu$ is the dynamic viscosity.

Equations (4) and (5) are substituted into equations (1), (2), and (3), and nondimensionalized by introducing the following characteristic scales (where the primed variables are dimensionless):

$$
\begin{gathered}
x_{i}=D x_{i}^{\prime} ; \quad u_{i}=U_{0} \dot{u_{i}} ; \quad T=\left(T_{b}-T_{0}\right) \theta+T_{0} ; \quad t=t^{\prime} D / U_{0} ; \\
E=\frac{H D^{2}}{\kappa\left(T_{b}-T_{0}\right)} ; \quad P=\frac{\mu_{r} U_{0}}{D} P^{\prime} ; \quad U_{0}=\kappa / D^{\prime}
\end{gathered}
$$

where $T_{b}$ and $T_{0}$ are the temperatures on the bottom and top boundaries, respectively; $D$ is the thickness of the box; $\theta$ is the nondimensional temperature; $\mu_{r}$ is a reference dynamic viscosity, which is chosen as the viscosity appropriate for the temperature on the bottom boundary for cases with temperature-dependent viscosity and otherwise is chosen as the background viscosity. The model and material parameters are listed in Table 1. The nondimensional equations are (primes have been dropped for simplicity)

$$
\begin{aligned}
& \sigma_{i j, j}+\left(R_{a} \theta-\sum_{k} R_{b}^{k} \Gamma_{k}\right) \delta_{i 2}=0 ; \\
& u_{i, i}=0 ;
\end{aligned}
$$

Table 1. Physical and Geometrical Model Parameters

\begin{tabular}{lc}
\multicolumn{1}{c}{ Parameter } & Value \\
\hline Thickness of the box $D$ & $1.5 \times 10^{6} \mathrm{~m}$ \\
Thickness of the lithosphere & $1.0 \times 10^{5} \mathrm{~m}$ \\
Temperature contrast $T_{\mathrm{b}}-T_{\circ}$ & $2000 \mathrm{~K}$ \\
Reference density $\rho_{\mathrm{o}}$ & $3.3 \times 10^{3} \mathrm{kgm}^{-3}$ \\
Thermal diffusivity $\mathrm{K}$ & $10^{-6} \mathrm{~m}^{2} \mathrm{~s}^{-1}$ \\
Acceleration of gravity $g$ & $10 \mathrm{~m} \mathrm{~s}^{-2}$ \\
Thermal expansion $\alpha$ & $2.0 \times 10^{-5} \mathrm{~K}^{-1}$ \\
Thermal Rayleigh number $R_{a}$ & $10^{6}$ \\
Equilibrium depth for the & $4.1 \times 10^{5} \mathrm{~m}$ \\
$\quad$ exothermic phase change & $6.7 \times 10^{5} \mathrm{~m}$ \\
Equilibrium depth for the & $1200 \mathrm{~K}$ \\
endothermic phase change & \\
Equilibrium temperature for & \\
the exothermic phase change & \\
Equilibrium temperature for & \\
the endothermic phase change & $1000 \mathrm{~K}$ \\
Bottom heating & \\
Internal heating & \\
Density jumps across the & \\
phase change boundaries & \\
Phase transition widths $d$ & \\
\hline &
\end{tabular}

where in equation (7), the hydrostatic pressure has been eliminated; $R_{a}$ and $R_{b}^{k}$ are the thermal Rayleigh number and phase change Rayleigh number for the $k$ th phase change, respectively. Their definitions are

$$
\begin{aligned}
& R_{a}=\frac{\rho_{0} g \alpha\left(T_{b}-T_{0}\right) D^{3}}{\kappa \mu_{r}} \\
& R_{b}^{k}=\frac{\Delta \rho_{k} g D^{3}}{\kappa \mu_{r}}
\end{aligned}
$$

The approach to incorporate phase changes is similar to that of Richter [1973] and Christensen and Yuen [1985]. We define an "excess pressure" as

$$
\pi_{k}=P-P_{0}-\gamma_{k} T \text {, }
$$

where $\gamma_{k}$ and $P_{0}$ are the Clapeyron slope and phase change pressure at zero degree temperature for the $k$ th phase change. After nondimenionalizing pressure terms and $\gamma_{k}$ by $\rho_{0} g D$ and $\rho_{0} g D /\left(T_{b}-T_{0}\right)$, respectively, and ignoring the nonhydrostatic pressure, the nondimensional "excess pressure" can be written as

$$
\pi_{k}=1-d_{r k}-x_{2}-\gamma_{k}\left(\theta-\theta_{r k}\right),
$$

where $\gamma_{k}, d_{r k}$, and $\theta_{r k}$ are the nondimensional Clapeyron slope, reference phase transition depth, and reference phase transition temperature for the $k$ th phase change, respectively. For the $410-\mathrm{km}$ and $670-\mathrm{km}$ phase changes, $d_{r k}$ are 0.273333 and 0.446667 , respectively. $\theta_{r k}$ vary from case to case depending on heating modes; $\theta_{n k}$ are 0.5 for noninternal heating cases, and for internal heating cases, $\theta_{n k}$ are 0.6 and 0.7 for the $410-\mathrm{km}$ and $670-\mathrm{km}$ phase changes, respectively. The dimensionless phase change function for the $k$ th phase change, $\Gamma_{k}$, is then defined as

$$
\Gamma_{k}=\frac{1}{2}\left(1+\tanh \frac{\pi_{k}}{d}\right),
$$


where $d$, the dimensionless phase transition width, is 0.025 (which scales to $37.5 \mathrm{~km}$ ) for all the phase changes in this study.

Plates are simulated either through the material property method or an imposed surface velocity. For the material property method, a $100-\mathrm{km}$-thick layer near the surface is assigned a viscosity $10^{3}$ times the interior viscosity, and this region represents a plate (Figure 1). Two margins of the plate have a viscosity 0.1 time the interior viscosity (Figure 1 ). The low viscosity for the two margins and the high viscosity for the plate are designed to mimic a stress- and temperaturedependent rheology. Plates with different length can be obtained by varying the aspect ratio of the box. To use temperature- and stress-dependent rheology is computationally costly, since the stiffness matrix would need to be factorized numerous times for each time step. However, for the material property method, the factorization of the stiffness matrix is only needed once at the beginning of each calculation; therefore the material property method is an efficient and effective method for modeling plates, and we will use this method to mainly study effects of plates on phase change dynamics.

Temperature-dependent viscosity is necessary to investigate the effects of stiff subducted slabs. While temperature-dependent viscosity gives rise to both stiff plates and slabs, it causes plates to become immobile [e.g., Gurnis, 1989]. Therefore imposing a constant surface velocity is necessary to have a realistic plate [Davies, 1988a]. By imposing a constant surface velocity in temperature-dependent viscosity models, plates and stiff slabs can be well simulated. We have found that the imposed surface velocity method does not alter the dynamics, as long as the imposed surface velocity is equal to the average plate velocity determined from the material property method. The temperature dependence of viscosity is defined as

$$
\mu=\exp \left(\frac{c_{1}}{\theta+c_{2}}-\frac{c_{1}}{1+c_{2}}\right)
$$

where constants $c_{1}$ and $c_{2}$ determine both the range of viscosity variations within the fluid and the viscosity drop across top thermal boundary layer. In this study, two sets of constants $c_{1}$ and $c_{2}$ have been used. Both sets of constants limit the viscosity to vary from 1 to $10^{3}$ as nondimensional temperature changes from 1 to 0 , but the viscosity drops across the top thermal boundary layer are different (Figure 2).

Isothermal boundary conditions are applied on the top and bottom boundaries; reflecting boundary conditions are used for

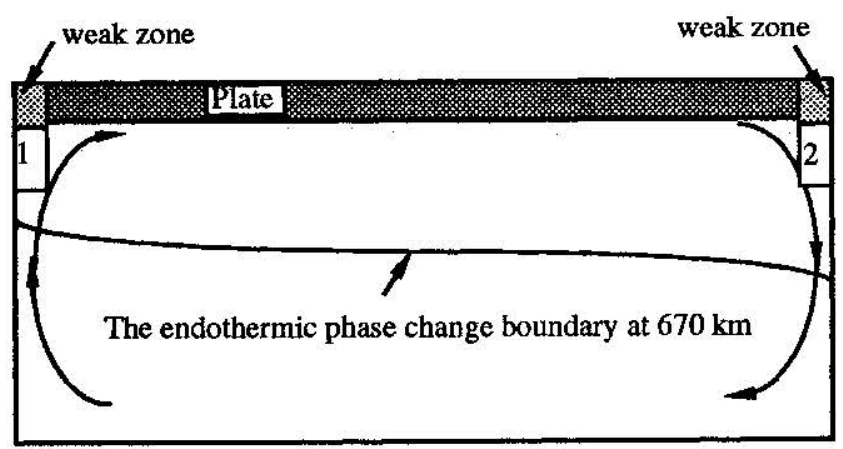

Figure 1. Model setup using the material property method. The numbers represent regions in which mean temperatures are measured. Regions 1 and 2 overlap the weak zones.

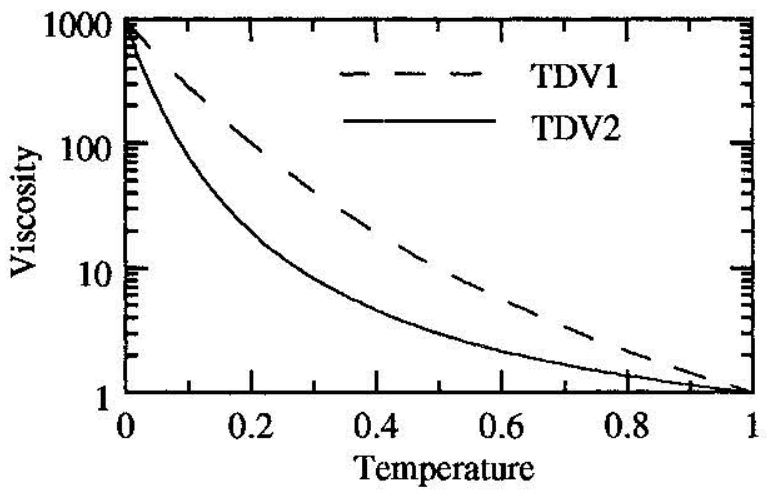

Figure 2. The temperature dependence of viscosity. For TDV1, $c_{1}$ and $c_{2}$ are 13.82 and 1.0, respectively; for TDV2, $c_{1}$ and $c_{2}$ are 1.987 and 0.2332 , respectively.

the side walls; and free slip boundary conditions are applied on the top and bottom boundaries except for the cases with imposed surface velocity. The initial conditions depend on the aspect ratio. For a given aspect ratio, a case with no phase change is run with an initial condition,

$$
\theta\left(x_{1}, x_{2}\right)=1-x_{2}+\varepsilon \cos \left(\pi \frac{x_{1}}{L}\right) \sin \left(\pi x_{2}\right),
$$

to check whether a "plate-like" surface deformation can be obtained using the material property method, where in equation (16), $\varepsilon$, the perturbation, is $0.01 ; L$ is the aspect ratio or the plate length. For all the cases with phase changes, the initial conditions are taken from the last frame of that run with no phase changes for the corresponding aspect ratio.

The equations are solved with a finite element code: ConMan [King et al., 1990]. A penalty formulation is used to solve the momentum equation and to enforce incompressibility [Hughes, 1987]. The energy equation is solved with a streamline upwind Petrov-Galerkin method [Brooks, 1981]. There are 80 elements in the vertical direction and 80 to 250 elements in the horizontal direction, depending on the aspect ratio of the box. By refining meshes, sufficient resolution is acquired in plates, plumes, and phase transition zones. There are more than seven elements to resolve the top and bottom thermal boundaries and the upwelling and downwelling limbs near the side walls. We have compared results from our ConMan code with phase changes with results from a finite difference code discussed by Weinstein [1993]; Nusselt numbers for test cases agree well between the codes with differences of less than $1 \%$.

It should be pointed out that due to computational limitations our models are simplified in both material property and geometry, compared to Earth's mantle. The threedimentional spherical geometry of Earth may give rise to different forms of upwellings and downwellings from those observed in two-dimesional models, which may affect the ability of mass exchange across the phase change boundary [Tackley et al., 1993]. The thickness of the box in our models is only half the depth of the mantle; the small thickness of the box may reduce the potential of thermal buoyancy and thus affects the phase change dynamics. A more realistic rheology of Earth's mantle may result in a larger viscosity contrast than $10^{3}$ in our models, and the larger viscosity contrast would give rise to stiffer plates and slabs than those in our models. However, we think that the effects of tectonic plates and stiff subducted slabs on phase change dynamics, which are 
demonstrated in our models, are essential for understanding the thermal and chemical structure of Earth's mantle.

\section{Measures of Temporal and Spatial Dependencies}

Several measures are made to study the dynamics of slab penetration and the layering of convection. First, a slab penetration velocity $V_{s}$ is defined as

$$
V_{s}=\frac{1}{\Delta x} \int_{L-\Delta x}^{L}\left|u_{2}^{e n d o}\left(x_{1}\right)\right| d x_{1} \text {, }
$$

where $\Delta x$ is 0.0667 (or $100 \mathrm{~km}$ ) and $u_{2}^{\text {endo }}$ is the vertical velocity on the $670-\mathrm{km}$ endothermic phase change boundary. With the initial conditions used, a strong downwelling limb (subducting slab) is assured on the right side of the box (Figure 1). Second, a mass flux through the entire endothermic phase change boundary is defined as

$$
M_{v}=\int \Gamma_{\Gamma_{e n d o}}\left|u_{n}^{e n d o}(l)\right| d l,
$$

where the line integral is taken along the endothermic phase change boundary, $\Gamma_{\text {endo }}$, and $u_{2}^{\text {endo }}$ is the normal velocity on $\Gamma_{\text {endo }}$. A maximum horizontal mass flux is defined as

$$
M_{h}=\max _{0 \leq x_{1} \leq L}\left[\int_{0}^{1}\left|u_{1}\left(x_{2}\right)\right| d x_{2}\right],
$$

Since $M_{v}$ should be zero for perfect layered convection but close to $M_{h}$ for a convection cell which completely penetrates the phase change, the ratio of $M_{v}$ to $M_{h}$ measures the degree of layering. Third, a parameter $R_{s}$ is defined as the fraction during which slab penetration velocity $V_{s}$ is more than $80 \%$ of plate velocity $U_{p}$, where $U_{p}$ is the average surface velocity. Fourth, average temperatures in upwelling, $T_{u}$, and downwelling, $T_{d}$, are computed (see Figure 1 for the regions in which the averages are computed). The differential temperature, $T_{u}-T_{d}$, is a measure of the largest-scale thermal buoyancy.

Besides these four measures, we also compute mean temperature $T_{m}$, surface heat flux $F$, internal heating rate $\xi$, plate velocity $U_{p}$, and plateness $P$. The procedures to calculate surface heat flux and internal heating rate are identical to those given by Zhong and Gurnis [1993]. Following Weinstein and Olson [1992], plateness measures how well a model plate achieves "plate-like" behavior:

$$
P=\frac{1}{\pi-2}\left(\pi-\frac{1}{U_{p}} \int_{0}^{l}\left|\frac{d u_{1}\left(x_{1}, 1\right)}{d x_{1}}\right| d x_{1}\right),
$$

where $u_{1}\left(x_{1}, 1\right)$ is the surface horizontal velocity. $P$ is a number between 0 and $1 ; P$ is 1 when the surface velocity becomes a box-car function (i.e., a plate) and 0 for a sinusoidal function surface velocity. However, caution must be exercised in using this characterization because minor kinks in the surface horizontal velocity profile, which may be visually insignificant, can significantly reduce $P$.

\section{Results and Discussions}

Plates affect phase change dynamics in two ways. First, by inducing a predominantly plate-scale flow, plates control the large-scale buoyancy distribution and thus affect the mass exchange across the endothermic phase change boundary.
Second, by retaining the negative buoyancy of a cooling thermal boundary layer, plates of different sizes control the amount of negative buoyancy prior to the intersection of the phase boundary. Subducted slabs are stiffer than ambient mantle, due to the lower temperature of slabs, and stiff slabs tend to retain their integrity and thus have a larger chance to penetrate into the lower mantle. These effects of plates and stiff subducted slabs on phase change dynamics are evident in our numerical models for a wide range of parameters. In what follows, we will first present calculations incorporating plates of large size relative to the box depth using the material property method; second, we show the effects of plates on endothermic phase change dynamics and on multiple phase change dynamics; finally, we study the effects of stiff slabs on slab penetration using temperature-dependent viscosity.

\section{Simulation of Plates Through the Material Property Method}

Plates are simulated with a material property method. With the material property method, the surface velocity of plates with a length comparable to the box height has been shown to be "plate-like" [Gurnis, 1989; King et al., 1992]; however, no similar results have yet been reported for large size plates.

Models with plates whose lengths are $1,2,3$ and 5 times the box height (cases $1,2,3$, and 4, respectively in Table 2) show that the material property method can yield a "platelike" surface velocity for plates with $P$ greater than 0.9 (Table 2 ). These cases have no phase change and are heated entirely from below (Table 2) with $R_{a}=10^{6}\left(R_{a}\right.$ is $10^{6}$ for all the cases in this study unless otherwise specified). Plate-scale flow and thermal structure predominate and "plate-like" behavior is well simulated even for an $L$ of 5 (representative surface velocity profiles, temperature fields and stream functions for cases with $L=1,3$, and 5 are shown in Figure 3). Plates continue to cool until they reach the weak margins where the cold fluid plunges into the fluid interior.

As $L$ increases, we observe that time-averaged vertical and horizontal mass flux $\left\langle M_{v}\right\rangle$ and $\left\langle M_{h}\right\rangle$, plate velocity $\left\langle U_{p}\right\rangle$, and slab penetration velocity $\left\langle V_{s}\right\rangle$ in general increase (Table 2). The amount of increase is less than $50 \%$, as $L$ increases from 1 to 5 (Table 2). The ratios of $\left\langle M_{v}\right\rangle$ to $\left\langle M_{h}\right\rangle$ are all slightly larger than 1 with a maximum of 1.25 for case 2 (Table 2), and this has two implications: (1) a single-cell flow organized by the plate predominates, independent of plate length; (2) the ratio of $\left\langle M_{v}\right\rangle$ to $\left\langle M_{h}\right\rangle$ should be close to 1 for unlayered convection with plates. We also observe that as $L$ increases, the time-averaged heat flux $F$ through the surface decreases, but the mean temperature $T_{m}$ and the temperature difference between upwelling and downwelling $\left\langle\delta T_{h}\right\rangle$ (i.e., $T_{u}-T_{d}$ ) generally increase (Table 2 ). When significant internal heating is included (cases $5,6,7$, and 8 for $L=1,2,3$, and 5 , respectively, in Table 2), plate-scale flow predominates, and plates are well-simulated (Table 2), similar to the no internal heating cases. However, $\left\langle M_{v}\right\rangle,\left\langle M_{h}\right\rangle$, and $\left\langle U_{p}\right\rangle$ are significantly smaller than those for no internal heating cases for $L=1,2$, and 3 .

The plate-scale flow structures (e.g., Figures $3 b$ and $3 c$ ) are similar to those with prescribed surface velocity and temperature-dependent viscosity [Davies, 1988a] but are significantly different from models with no plates in which flow structures are of much smaller wavelength (e.g., Figure 4a shows representative temperature and flow fields for case 9 with isoviscous flow (Table 2); case 9 is identical to case 3 
Table 2. Model Parameters and Statistics of Models

\begin{tabular}{|c|c|c|c|c|c|c|c|c|c|c|c|c|c|c|c|}
\hline Case & Mode & $L$ & $E$ & $\gamma_{1}$ & $\gamma_{2}$ & $R_{s}$ & $\left\langle M_{v}\right\rangle$ & $<M_{h}>$ & $\left\langle V_{s}\right\rangle$ & $<U_{p}>$ & $<\delta T_{h}>$ & $P$ & $F$ & $\xi \%$ & $T_{m}$ \\
\hline 1 & MP & 1 & 0 & - & - & 1.0 & $545(9)$ & $545(11)$ & $1450(16)$ & $697(5)$ & 0.30 & 0.91 & 20.0 & 0 & 0.52 \\
\hline 2 & MP & 2 & 0 & - & - & 1.0 & $619(40)$ & $494(39)$ & $1665(89)$ & $703(55)$ & 0.42 & 0.95 & 14.8 & 0 & 0.54 \\
\hline 3 & MP & 3 & 0 & - & - & 1.0 & $682(63)$ & $572(44)$ & $1928(126)$ & $710(71)$ & 0.41 & 0.95 & 12.3 & 0 & 0.59 \\
\hline 4 & $\mathbf{M P}$ & 5 & 0 & - & - & 1.0 & $776(73)$ & $659(60)$ & $2167(177)$ & $790(77)$ & 0.45 & 0.93 & 10.2 & 0 & 0.61 \\
\hline 5 & $\mathrm{MP}$ & 1 & 8 & - & - & 1.0 & $398(51)$ & $394(58)$ & $1451(72)$ & $562(47)$ & 0.31 & 0.91 & 19.5 & 24 & 0.61 \\
\hline 6 & MP & 2 & 8 & - & - & 1.0 & $493(41)$ & $474(39)$ & $1757(107)$ & $614(74)$ & 0.42 & 0.94 & 15.2 & 26 & 0.64 \\
\hline 7 & MP & 3 & 8 & - & - & 1.0 & $575(51)$ & $536(49)$ & $1964(129)$ & $622(70)$ & 0.42 & 0.93 & 13.1 & 26 & 0.68 \\
\hline 8 & $\mathrm{MP}$ & 5 & 8 & - & - & 1.0 & $773(28)$ & $704(28)$ & $2396(88)$ & $782(30)$ & 0.48 & 0.92 & 11.6 & 23 & 0.70 \\
\hline 9 & $\mathrm{CV}$ & - & 0 & - & - & - & $1331(412)$ & $693(199)$ & - & $528(256)$ & - & - & 17.3 & 0 & 0.50 \\
\hline 10 & $\mathrm{CV}$ & - & 0 & - & -0.17 & - & 101(32) & $301(30)$ & - & $340(54)$ & - & - & 9.5 & 0 & 0.52 \\
\hline 11 & MP & 1 & 0 & - & -0.17 & 0.05 & $34(18)$ & $212(30)$ & $113(85)$ & $300(44)$ & 0.33 & 0.90 & 10.5 & 0 & 0.54 \\
\hline 12 & $\mathrm{MP}$ & 2 & 0 & - & 0.17 & 0.38 & $212(210)$ & $288(196)$ & $350(414)$ & $398(220)$ & 0.42 & 0.81 & 11.2 & 0 & 0.60 \\
\hline 13 & $\mathrm{MP}$ & 3 & 0 & - & -0.17 & 0.48 & $333(144)$ & $395(169)$ & $502(502)$ & $466(174)$ & 0.47 & 0.92 & 10.2 & 0 & 0.62 \\
\hline 14 & MP & 5 & 0 & - & -0.17 & 0.48 & $465(120)$ & $471(120)$ & $518(445)$ & $541(124)$ & 0.56 & 0.94 & 9.01 & 0 & 0.64 \\
\hline 15 & MP & 1 & 8 & - & -0.17 & 0.00 & $26(2)$ & $188(6)$ & $100(11)$ & $325(4)$ & 0.33 & 0.89 & 12.7 & 55 & 0.66 \\
\hline 16 & MP & 2 & 8 & - & -0.17 & 0.32 & $195(172)$ & $270(157)$ & $319(411)$ & $426(182)$ & 0.49 & 0.84 & 14.4 & 42 & 0.72 \\
\hline 17 & MP & 3 & 8 & - & -0.17 & 0.43 & $284(118)$ & $329(137)$ & $461(482)$ & $469(146)$ & 0.52 & 0.92 & 12.7 & 41 & 0.76 \\
\hline 18 & MP & 5 & 8 & - & -0.17 & 0.48 & $410(123)$ & $423(150)$ & $532(534)$ & $516(129)$ & 0.57 & 0.92 & 10.9 & 43 & 0.79 \\
\hline 19 & MP & 1 & 8 & - & -0.19 & 0.01 & $21(8)$ & $183(28)$ & $75(43)$ & $316(5)$ & 0.31 & 0.91 & 11.5 & 61 & 0.66 \\
\hline 20 & MP & 2 & 8 & - & -0.19 & 0.00 & $47(24)$ & $234(40)$ & $8(3)$ & $362(18)$ & 0.52 & 0.92 & 11.0 & 65 & 0.70 \\
\hline 21 & $\mathrm{MP}$ & 3 & 8 & - & 0.19 & 0.30 & $239(134)$ & $294(140)$ & $319(434)$ & $436(154)$ & 0.54 & 0.90 & 12.6 & 43 & 0.76 \\
\hline 22 & MP & 5 & 8 & - & -0.19 & 0.33 & $333(92)$ & $358(128)$ & $333(480)$ & $466(108)$ & 0.61 & 0.91 & 10.8 & 48 & 0.79 \\
\hline 23 & MP & 3 & 8 & - & -0.12 & 1.0 & $379(66)$ & $369(46)$ & $965(129)$ & $564(90)$ & 0.45 & 0.93 & 13.0 & 33 & 0.72 \\
\hline 24 & $\mathrm{MP}$ & 3 & 8 & - & -0.15 & 0.51 & $321(130)$ & $355(146)$ & $564(484)$ & $494(155)$ & 0.48 & 0.91 & 12.8 & 37 & 0.76 \\
\hline 25 & MP & 3 & 8 & - & -0.21 & 0 & $96(36)$ & $265(60)$ & $14(9)$ & $396(37)$ & 0.65 & 0.93 & 12.1 & 56 & 0.73 \\
\hline 26 & MP & 1 & 8 & 0.17 & -0.17 & 0.19 & $133(195)$ & $243(166)$ & $276(383)$ & $431(249)$ & 0.25 & 0.80 & 17.5 & 32 & 0.61 \\
\hline 27 & $\mathbf{M P}$ & 2 & 8 & 0.17 & -0.17 & 0.31 & $286(257)$ & $342(230)$ & $451(581)$ & $505(331)$ & 0.35 & 0.81 & 14.3 & 29 & 0.68 \\
\hline 28 & MP & 3 & 8 & 0.17 & -0.17 & 0.43 & $409(198)$ & $426(180)$ & $567(599)$ & $616(252)$ & 0.39 & 0.90 & 13.3 & 32 & 0.70 \\
\hline 29 & $\mathrm{MP}$ & 5 & 8 & 0.17 & -0.17 & 0.54 & $541(171)$ & $508(143)$ & $697(612)$ & $683(171)$ & 0.43 & 0.90 & 11.4 & 29 & 0.72 \\
\hline 30 & TDV & - & 8 & - & -0.17 & - & $47(10)$ & $170(26)$ & - & $74(4)$ & - & - & 8.71 & 88 & 0.85 \\
\hline 31 & MP & 3 & 8 & - & - & 1.0 & $560(29)$ & $522(13)$ & $1925(53)$ & 622 & 0.52 & 1.0 & 13.0 & 30 & 0.68 \\
\hline 32 & MF & 3 & 8 & - & -0.17 & 0.42 & $294(60)$ & $334(105)$ & $458(440)$ & 469 & 0.60 & 1.0 & 12.5 & 39 & 0.76 \\
\hline 33 & IDVI & 3 & 8 & - & - & 1.0 & $409(48)$ & $374(20)$ & $948(22)$ & 622 & 0.67 & 1.0 & 14.1 & 40 & 0.77 \\
\hline 34 & IDVI & 3 & 8 & - & -0.17 & 0.99 & $228(45)$ & $244(35)$ & $444(17)$ & 469 & 0.74 & 1.0 & 13.5 & 49 & 0.84 \\
\hline 35 & TDVI & 3 & 8 & - & -0.21 & 0.26 & $93(7)$ & $197(9)$ & $313(13)$ & 396 & 0.81 & 1.0 & 13.1 & 57 & 0.83 \\
\hline 36 & TDV2 & 3 & 8 & - & 0.17 & 0.59 & $263(57)$ & $276(79)$ & $417(192)$ & 469 & 0.71 & 1.0 & 13.5 & 51 & 0.83 \\
\hline
\end{tabular}

In the column of mode, MP, CV, MPI, TDV1, and TDV2 stand for material property plate, constant viscosity, material property with imposed surface velocity plate, temperature-dependent viscosity with TDV1 (Figure 2), and temperaturedependent viscosity with TDV2 (Figure 2), respectively; the numbers in parentheses are the standard deviations.

except the rheology). Since multicell convection (i.e., smallscale flow structure) characterizes the flow for the isoviscous flow case (case 9), the ratio of $\left\langle M_{v}\right\rangle$ to $\left\langle M_{h}\right\rangle$ for case 9 is about twice larger than that for case 3 in which a single-cell convection characterizes the flow (Table 2).

\section{Endothermic Phase Change Dynamics With a Plate}

Inclusion of an endothermic phase change at $670 \mathrm{~km}$ depth may lead to layered convection if the Clapeyron slope and density contrast across the phase change are sufficiently large [Christensen and Yuen, 1984; 1985]. The critical Clapeyron slope for layered convection may decrease with increasing Rayleigh number $R_{a}$ [Christensen and Yuen, 1985; Zhao et al., 1992]. In this section, we shall focus on the effects which plates have on thermal convection with phase changes.

In order to better examine the effect of a plate on phase change dynamics, an isoviscous flow (case 10 in Table 2) with an endothermic phase change is computed; the inclusion of the phase change is the only difference between this case (case 10) and the previous isoviscous flow case (case 9) (Table 2). For this entirely bottom heated case, the thermal Rayleigh number $R_{a}$, phase change Rayleigh number $R_{b}$, and nondimensional Clapeyron slope $\gamma$ are $10^{6}, 2 \times 10^{6}$, and -0.17 (Table 2), respectively. In this study, $R_{b}$ for either exothermic or endothermic phase changes is always twice that of $R_{a}$, i.e., $2 \times 10^{6}$, unless otherwise specified. The density jump across the phase boundary and the Clapeyron slope, after scaling with the material parameters in Table 1 , are $8 \%$ and $-4.21 \mathrm{MPa} \mathrm{K}^{-1}$, respectively. For this isoviscous case (case 10 ), the endothermic phase change results in clearly layered convection, which is evident through representative temperature and stream functions (Figure $4 \mathrm{~b}$ ) and a ratio of $\left\langle M_{v}\right\rangle$ to $\left\langle M_{h}\right\rangle$ which is only about 0.3 (Table 2). Since an endothermic phase change retards convection, $\left\langle M_{v}\right\rangle$ and $\left\langle M_{h}\right\rangle$ are much smaller, compared with those without the phase change (Table 2). It should be pointed out that for the $R_{a}$, Clapeyron slope, and density jump used in case 10 , thermal convection was also reported to be predominantly layered in an isoviscous flow model in a unit aspect ratio box [Christensen and Yuen, 1985]. 

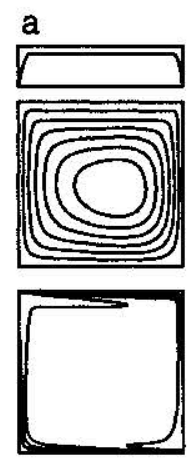

b
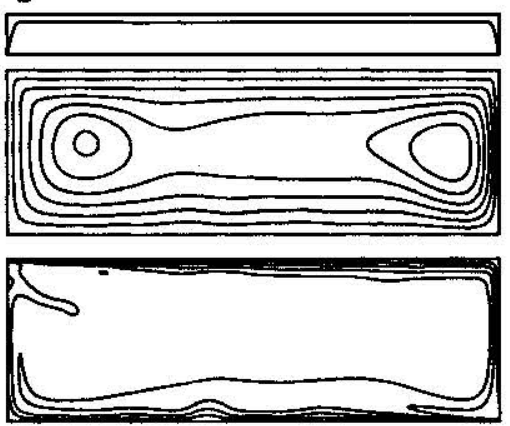

c
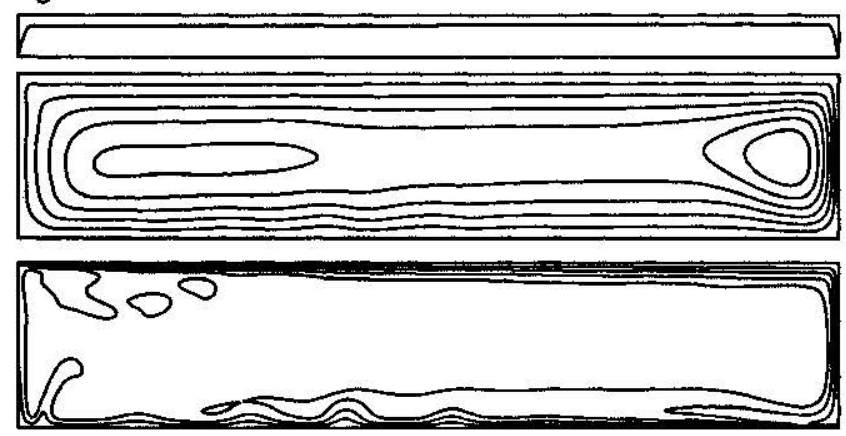

Figure 3. Representative temperature and stream functions for (a) case 1 with $L=1$, (b) case 3 with $L=3$, and (c) case 4 with $L=5$. In each part, the top panel is surface velocity, the middle panel is the stream function, and the bottom panel is the temperature. All three temperature fields have the same contours.

Models with the same $R_{a}, R_{b}, \gamma$, and internal heating parameter $E$ as those in case 10 but with a plate (cases 11,12 , 13, and 14 in Table 2 for $L=1,2,3$, and 5, respectively) show that plates exert a fundamental control on phase change dynamics. For $L=1$, the flow structures are characterized by two layered convection (Figure 5a) and differs significantly from those excluding any phase change (Figure 3a), indicating an important role of phase change in determining the thermal structure for $L=1$. The layered flow structures are similar to those of Christensen and Yuen [1985], suggesting that small plates do not significantly change the results of isoviscous flows. However, flows for models with long plates are characterized by plate-scale structure (Figures $5 \mathrm{~b}$ and $5 \mathrm{c}$ for representative temperature and flow fields for cases 13 and 14 with $L=3$ and 5 , respectively). These plate-scale thermal structures for long plates (Figures $5 \mathrm{~b}$ and $5 \mathrm{c}$ ) are very similar to those in flows including plates and lacking a phase change (e.g., Figures $3 b$ and $3 c$ ), but differ significantly from those in flows including a phase change and lacking plates (e.g., Figure $4 \mathrm{~b}$ for case 10). This suggests that for $L=3$ and 5, flow structures are primarily determined by the scale of plates not the phase change. The endothermic phase change has much larger effects on flow structures for models with small plates than for models of large plates, and for a large plate, the flow structures are predominantly controlled by the scales of the plate.

The phase change introduces an episodic time dependence. The effects of a phase change on the temporal evolution of mass flux $M_{\nu}$ across the phase boundary, slab penetration velocity $V_{s}$, and plate velocity $U_{p}$ also strongly depend on plate length. For $L=1$, convection is predominately layered, evident from the layered flow structures (Figure 5a). $V_{s}$ is always significantly smaller than plate velocity $U_{p}$ at any given time (Figure 6a), indicating insignificant slab penetration; $M_{v}$ is also considerably smaller than $M_{h}$, indicating layered convection and no significant mass exchange across the phase change boundary. For $L$ greater than $2, M_{v}$ becomes nearly as large as $M_{h} ; V_{s}$ is frequently much larger than plate velocity (Figures $6 \mathrm{~b}$ and $6 \mathrm{c}$ for cases 13 and 14 with $L=3$ and 5 , respectively), showing a diminished
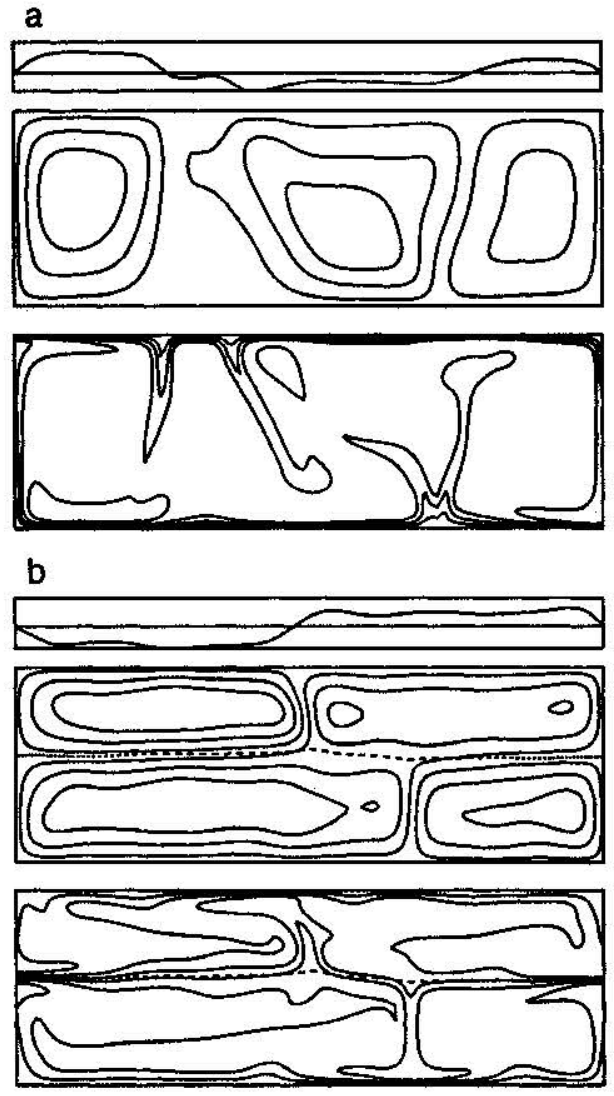

c
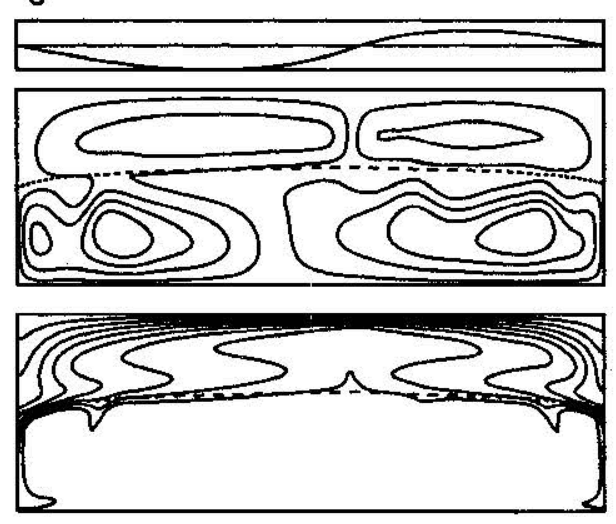

Figure 4. Representative temperature and stream functions for (a) isoviscous flow with no phase change (case 9), (b) isoviscous flow with an endothermic phase change (case 10), and (c) a temperature-dependent viscosity case with an endothermic phase change (case 30). The dashed lines represent the endothermic phase change boundary. For other details, see Figure 3. Note that six contours are used in Figures $4 a$ and $4 b$, but eight contours are used in Figure $4 c$ in order to visualize the weak thermal wisps in the lower layer. 
a
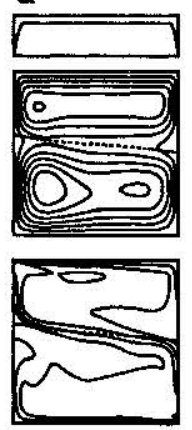

c
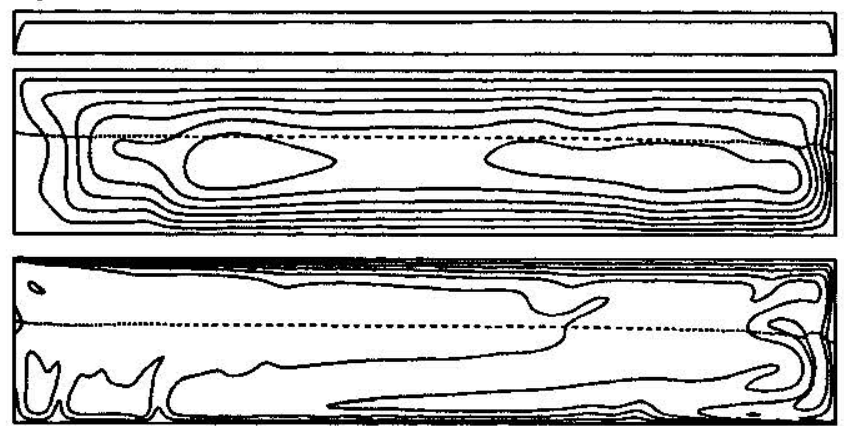

Figure 5. Representative temperature and stream functions for cases with an endothermic phase change and a plate of different length: (a) case 11 with $L=1$, (b) case 13 with $L=3$, and (c) case 14 with $L=5$. For other details, see Figure 4 .

degree of layered convection and intermittent slab penetration.

Increasing slab penetration and mass exchange across the phase boundary with $L$ is also evident through the time averaged quantities, $\left\langle M_{y}\right\rangle$ and $\left\langle V_{s}\right\rangle$. Both $\left\langle M_{v}\right\rangle$ and $\left\langle V_{s}\right\rangle$ increase rapidly with $L$ at a rate much larger than that for cases without a phase change (cases $1-4$ in Table 2). As $L$ increases, both $\left\langle M_{v}\right\rangle$ and $\left\langle V_{s}\right\rangle$ approach $\left\langle M_{h}\right\rangle$ and $\left\langle U_{p}\right\rangle$, respectively (Table 2 and Figures $7 \mathrm{a}$ and $7 \mathrm{~b}$ ). For $L=1$ (case 11), $\left\langle V_{s}\right\rangle$ is only about one third of $\left\langle U_{p}\right\rangle$, and the ratio of $\left\langle M_{v}\right\rangle$ to $\left\langle M_{h}\right\rangle$ is 0.16 (Table 2 and Figure $7 \mathrm{~b}$ ). For $L=5$ (case 14), $\left\langle V_{s}\right\rangle$ is 0.96 of $\left\langle U_{p}\right\rangle$, and the ratio of $\left\langle M_{v}\right\rangle$ to $\left\langle M_{h}\right\rangle$ is about 0.99 (Table 2 and Figure $7 \mathrm{~b}$ ), a ratio close to that expected from a dominantly single-cell convection with no phase change (e.g., case 4). A predominantly layered system of convection when $L=1$ (case 11) becomes essentially unlayered when $L$ is increased to 5 (case 14), indicating that the ability of the phase change to affect the style of convection diminishes as plate size increases.

For large $L$, slab penetration is influenced by an endothermic phase change, and $V_{s}$ is often much smaller than $U_{p} . V_{s}$ always has a very large fluctuation, independent of plate length (Figures $6 \mathrm{~b}$ and $6 \mathrm{c}$ ); the standard deviations of $V_{s}$ are always comparable to $\left\langle V_{s}\right\rangle$ and increase as $\left\langle V_{s}\right\rangle$ increases (values in parentheses in Table 2). Nevertheless, the effects of plate length $L$ on slab penetration are evident not only through the increase of $\left\langle V_{s}\right\rangle$ with $L$ but also through the increase of the time when $V_{s}$ is larger than $0.80 U_{p}$ normalized by total time $R_{s}$ (Table 2 and Figures $7 \mathrm{a}$ and $7 \mathrm{~b}$ ). $R_{s}$ increases from 0.05 when $L=1$ to 0.48 when $L=5$ (Table 2 and Figure $7 b$ ), indicating that slab penetration occurs more frequently for a long plate compared to a short plate.
$M_{v}, M_{h}$, and $U_{p}$ have smaller fluctuations as $L$ increases (Table 2 and Figures $6 \mathrm{~b}$ and $6 \mathrm{c}$ ); for $L$ greater than 2 , the standard deviations of $M_{v}, M_{h}$, and $U_{p}$ decrease as $\left\langle M_{v}\right\rangle$, $\left\langle M_{h}\right\rangle$, and $\left\langle U_{p}\right\rangle$ increase (Table 2). This is significantly different from the large fluctuations which are always observed for $V_{s}$. This suggests that the phase change may have less effects on $M_{v}, M_{h}$, and $U_{p}$ than on $V_{s}$, especially when $L$ is large. Moreover, while $V_{s}, M_{v}, M_{h}$, and $U_{p}$ usually simultaneously reach maxima and minima in their values, $V_{s}$ may occasionally be out of phase with $M_{v}, M_{h}$, and $U_{p}$ for $L$ $=3$ and 5 (Figures $6 \mathrm{~b}$ and $6 \mathrm{c}$ ). This could be due to $M_{v}, M_{h}$, and $U_{p}$ being controlled by different scale structure than $V_{s}$. While only plate-scale structures are capable of affecting $M_{v}$, $M_{h}$, and $U_{p}$, small-scale structures can influence $V_{s}$. For a larger $L$, the phase change has less effect on plate-scale structures, as discussed before; therefore $M_{v}, M_{h}$, and $U_{p}$ have smaller fluctuations which are presumably caused by the phase change. This explanation is supported by the correlations between plate-scale features including $M_{v}, M_{h}$,

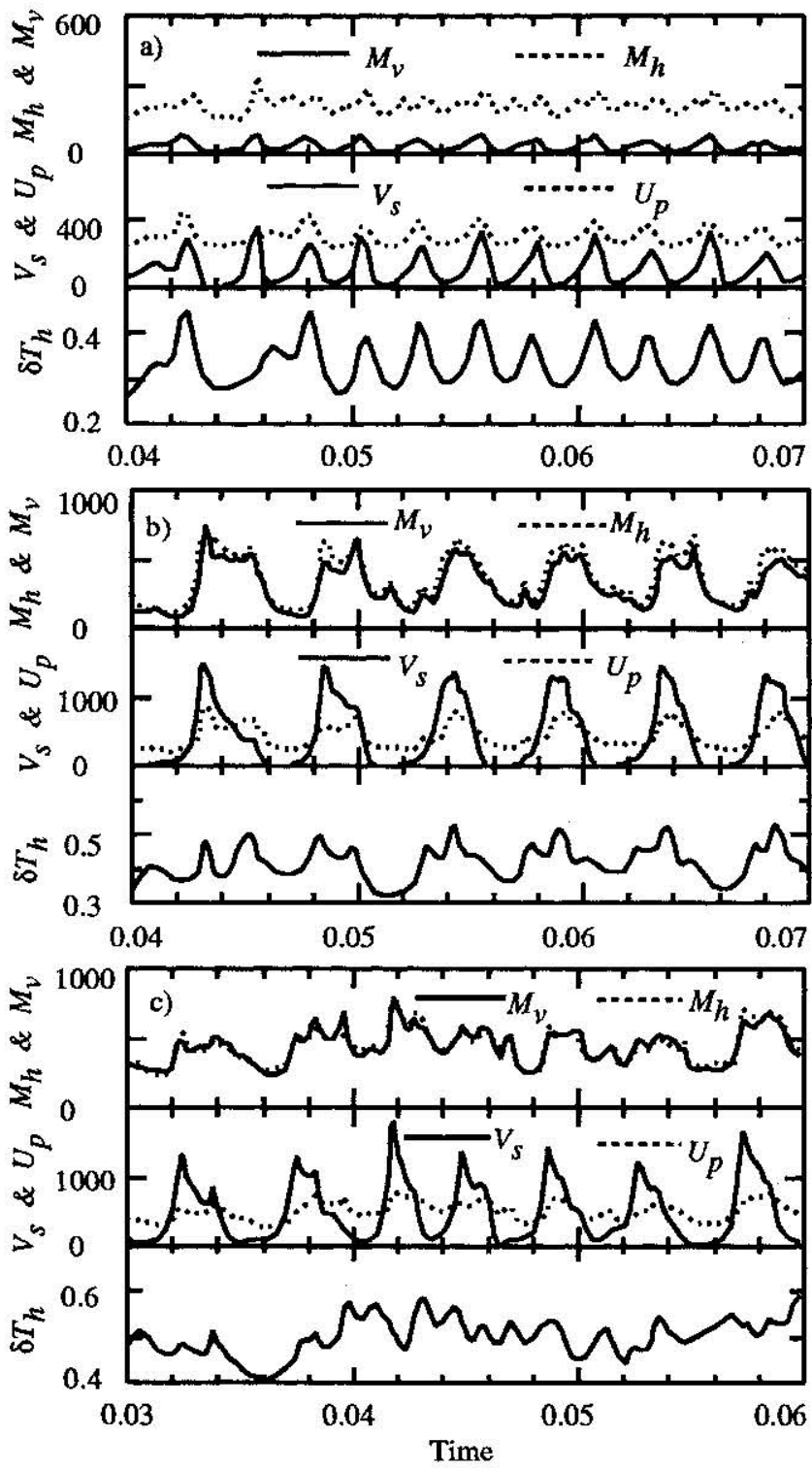

Figure 6. Time history of $M_{v}, M_{h}, V_{s}, U_{p}$, and $\delta T_{h}$ for (a) case $11,(b)$ case 13 , and (c) case 14 . The symbols are defined in the text. 

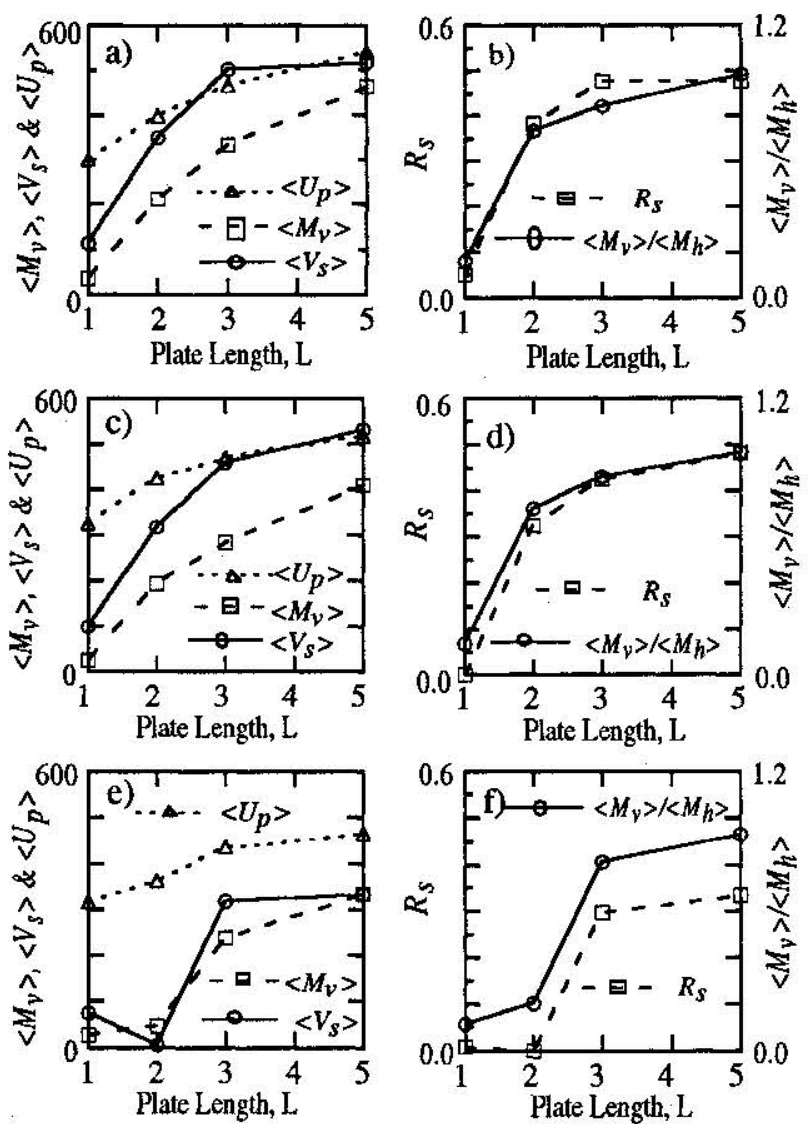

Figure 7. $\left\langle M_{v}\right\rangle,\left\langle V_{s}\right\rangle,\left\langle U_{p}\right\rangle$, the ratio of $\left\langle M_{v}\right\rangle$ to $\left\langle M_{h}\right\rangle$, and $R_{s}$, versus plate length for cases 11-14 (Figures 7a and $7 \mathrm{~b}$ ), cases 15-18 (Figures 7c and 7d), and cases 19-22 (Figures $7 \mathrm{e}$ and $7 \mathrm{f}$ ).

$U_{p}$, and $\delta T_{h}$, which measures the largest-scale thermal anomalies (Figures $6 \mathrm{a}, 6 \mathrm{~b}$, and $6 \mathrm{c}$ ). While $\delta T_{h}$ is usually correlated with $M_{v}, M_{h}$, and $U_{p}$, independent of $L, \delta T_{h}$ only has a correlation with $V_{s}$ for small $L$ (Figures $6 \mathrm{a}, 6 \mathrm{~b}$, and $6 \mathrm{c}$ ). Like $\left\langle U_{p}\right\rangle,\left\langle M_{v}\right\rangle$, and $\left\langle M_{h}\right\rangle$, time-averaged $\delta T_{h},\left\langle\delta T_{h}\right\rangle$, increases with $L$ (Table 2 and Figure $7 \mathrm{~b}$ ).

When a significant amount of internal heating is included (cases $15,16,17$, and 18 for $L=1,2,3$, and 5 , respectively), similar results are obtained. Four internally heated cases are identical to four bottom-heated cases except that the internal heating parameter $E$ is 8 (Table 2). For $L=1$ (case 15), $M_{y}$ and $V_{s}$ are much smaller than $M_{h}$ and $U_{p}$, respectively (Figure 8a), like the bottom heated case with $L=1$ (Figure 6 a for case 11 ), indicating layered convection. A steady state is now reached (Figure 8a), while convection is episodic for the bottom-heated case (Figure $6 a$ for case 11 ). As $L$ becomes larger than 1, convection becomes episodic; $V_{s}$ occasionally is much larger than $U_{p} ; M_{v}$ increases and approaches $M_{h}$ (Figures $8 \mathrm{~b}$ and $8 \mathrm{c}$ for cases 16 and 17 with $L=2$ and 3 , respectively). For $L=3, M_{v}$ essentially overlaps with $M_{h}$ (Figure $8 \mathrm{c}$ ), indicating insignificant layering for $L=3 . \delta T_{h}$ correlates with $M_{v}, M_{h}$ and $U_{p}$ (Figures $8 \mathrm{~b}$ and $8 \mathrm{c}$ ), but some secondary peaks in $\delta T_{h}$ often do not correlate with $V_{s}$ for large $L$ (Figure 8c). $\left\langle M_{v}\right\rangle,\left\langle V_{s}\right\rangle,\left\langle U_{p}\right\rangle$ and $R_{s}$ also increase substantially with $L$ (Figures $7 \mathrm{c}$ and $7 \mathrm{~d}$ and Table 2 ). Compared with those models with no internal heating, $\left\langle M_{v}\right\rangle$ decreases slightly and there is no significant change in $\left\langle V_{s}\right\rangle$ and $\left\langle U_{p}\right\rangle$ (Table 2 and Figures $7 \mathrm{a}$ and $7 \mathrm{c}$ ), suggesting a minor effect of internal heating on mass exchange and slab penetration across the phase change boundary, but heat flux $F$ and mean temperature $T_{m}$ due to the significant internal heating, both increase (Table 2). The internal heating rates, $\xi$, found a postpriori (Table 2), are $55 \%, 42 \%, 41 \%$, and $43 \%$ for $L=1$, 2,3 , and 5 , respectively (cases $15,16,17$, and 18 ).

Although an endothermic phase change has a diminishing effect on mass exchange across the phase boundary as $L$ increases, the phase change still causes slabs to bend and retards penetration even when $L=3$ and 5 (Figures $6 \mathrm{~b}, 6 \mathrm{c}$, and $8 \mathrm{c}$ for cases 13,14 , and 17 , respectively). A sequence of snapshots of temperature and flow fields for the internally heated case with $L=3$ (case 17) illustrate this process. At time A (time markings in Fig 8c), $M_{v}, V_{s}$, and $U_{p}$ peak (Figure $8 \mathrm{c}$ ); cold material within the downwelling penetrates the phase change (Figure 9a), accompanying a high horizontal thermal anomaly $\delta T_{h}$ (Figure $8 \mathrm{c}$ ). The slab penetration results in a low
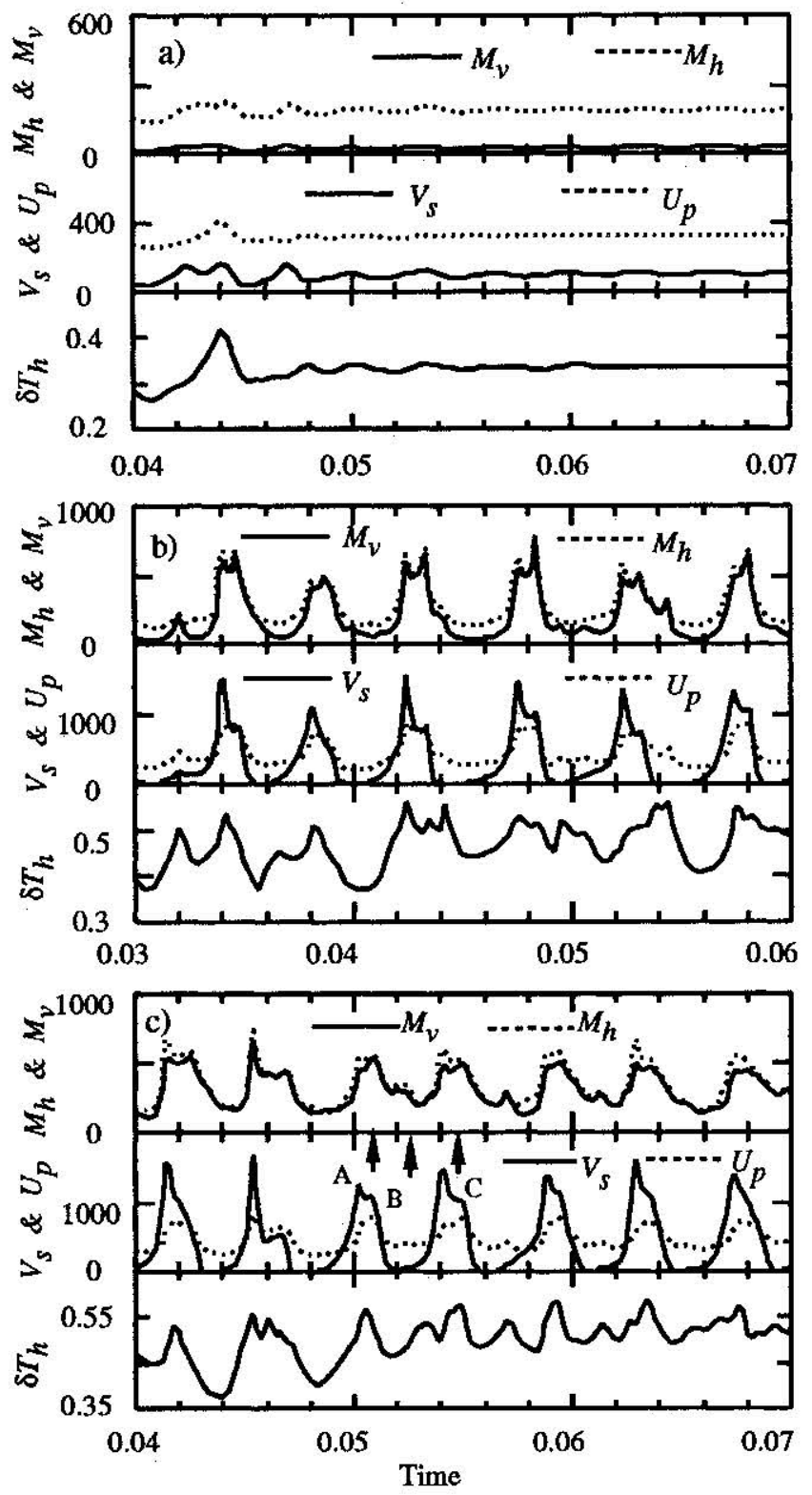

Figure 8. Time history of $M_{v}, M_{h}, V_{s}, U_{p}$, and $\delta T_{h}$ for cases with an endothermic phase change, internal heating, and a plate of different length: (a) case 15 with $L=1$, (b) case 16 with $L=2$, and (c) case 17 with $L=3$. 
a

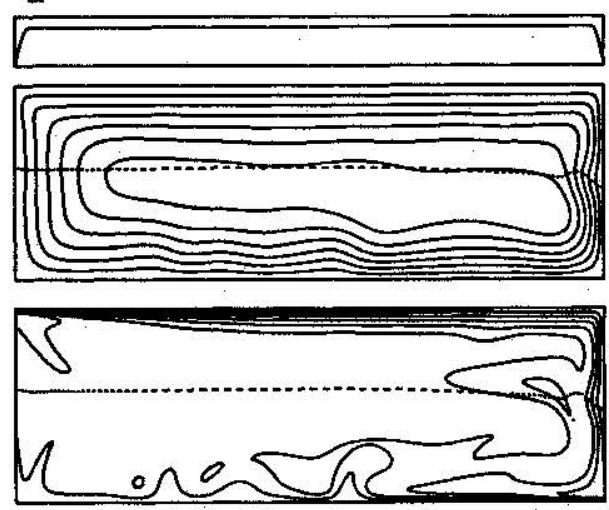

b
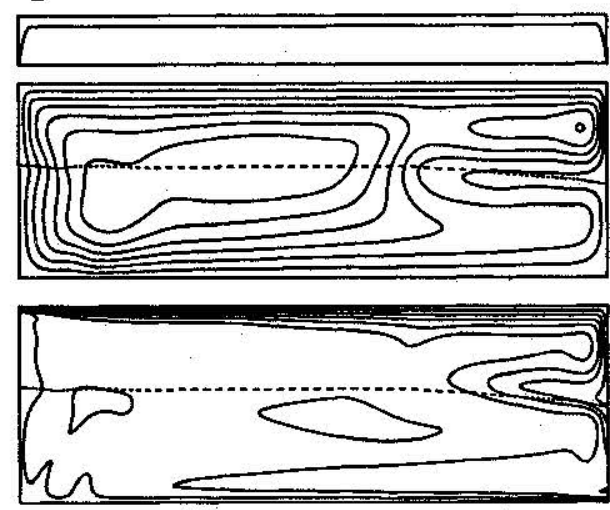

c
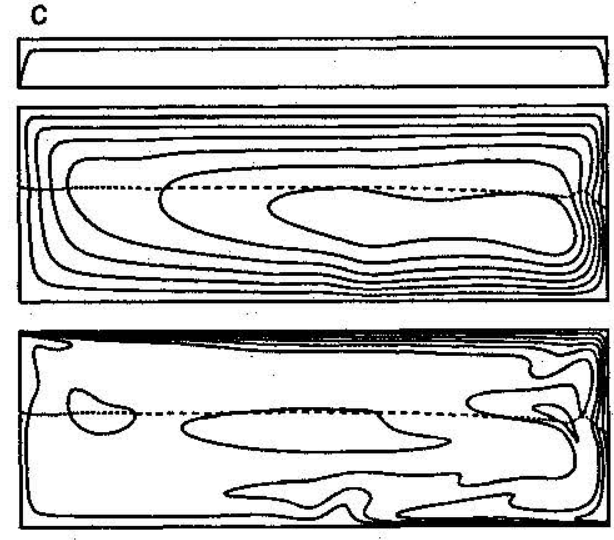

Figure 9. Temperature and stream functions at three instants in time, (a) $\mathrm{A}$, (b) $\mathrm{B}$, and, (c) $\mathrm{C}$ for case 17 (see the time markings in Figure 8c); for other details, see Figure 4.

temperature within the slab at the phase boundary with a large deflection of the boundary (Figure 9a), which gives rise to large phase change buoyancy. The increasing phase change buoyancy eventually causes the downwelling slab to bend and cold material to pile up above the phase change boundary by time $B$ (Figures $8 \mathrm{c}$ and $9 \mathrm{~b}$ ). At time $\mathrm{B}$, slab penetration velocity $V_{s}$ is at a minimum along with $M_{v}$ and $U_{p}$ (Figure $8 \mathrm{c}) ; \delta T_{h}$ is also at a minimum, indicating a small horizontal thermal anomaly (Figure 8c). $U_{p}$ is now significantly larger than $V_{s}$, so cold material tends to pile up above the phase boundary. As the slab bends horizontally, thermal buoyancy accumulates above the phase change boundary and phase change buoyancy decays because the higher temperature at the phase change gives less deflection of the phase boundary (Figure 9b). As the thermal buoyancy dominates at time $\mathrm{C}$, the downwelling slab penetrates again (Figure 9c), and $M_{v}, V_{s}$, and $U_{p}$ all reach their maximums (Figure $8 \mathrm{c}$ ). The horizontal thermal anomaly $\delta T_{h}$ also peaks. Even when slab penetration stops (Figure 9b), plate-scale flow structures still dominate, and there is significant mass exchange across the phase change boundary (Figure $8 \mathrm{c}$ ).

A steeper Clapeyron slope gives rise to larger deflections of phase boundaries, i.e., more phase change buoyancy; hence there is more resistance to slab penetration and mass exchange across the phase boundary. The effects of Clapeyron slope on thermal convection with plates are investigated in four cases with different plate lengths (cases 19, 20, 21, and 22 for $L=1$, 2,3 , and 5 , respectively), and these four cases only differ from the previous internally heated cases (cases 15, 16, 17, and 18) by an increased Clapeyron slope, $\gamma=-0.19$ (Table 2). The effects of plate length on phase change dynamics and flow structures for $\gamma=-0.19$ are similar to those for smaller $\gamma$. Convection remains episodic (Figures 10a, 10b, and 10c are time history plots for cases 20,21 , and 22 , with $L=2,3$, and 5 , respectively). As plate length $L$ increases, $\left\langle M_{v}\right\rangle$ and $\left\langle V_{s}\right\rangle$ increase and become as large as $\left\langle M_{h}\right\rangle$ and $\left\langle U_{p}\right\rangle$, respectively (Table 2 and Figure 7e), $R_{s}$ also increases with $L$ (Table 2 and Figure $7 \mathrm{f}$ ), but slab penetration is occasionally interrupted by the phase change for large $L$ (Figures $10 \mathrm{~b}$ and $10 \mathrm{c}$ for $L=3$ and 5). Predominant flow structures are of plate-scale wavelength (Figure $11 \mathrm{a}$ for representative temperature and stream functions for case 22 with $L=5$ ).

Compared with $\gamma=-0.17$ (cases 15-18), the steeper $\gamma$ results in more sluggish convection and a less efficient heat transfer. $R_{s},\left\langle U_{p}\right\rangle,\left\langle V_{s}\right\rangle,\left\langle M_{y}\right\rangle$, and heat flux $F$ all decrease (Table 2 and Figures 7c, 7d, 7e and 7f); none of these trends are surprising in light of past analysis [Christensen and Yuen, 1985]. Convection is layered not only for the case (case 19) with $L=1$ but also for $L=2$ (case 20) (Table 2 and Figures 10a, 7f, 11b). Layering vanishes for $L=3$ (Figure 10b for case 21) and the ratio of $\left\langle M_{v}\right\rangle$ to $\left\langle M_{h}\right\rangle$ increases rapidly to 0.81 (Figure $7 f$ and Table 2), which is only slightly smaller than that when $\gamma=-0.17$ for the same plate length (case 17 in Table 2 and Figure 7d). Convection for $L=2$ (Figure 11b) is characterized by "leaking" convection, as originally discovered by Christensen and Yuen [1985]; this is different from complete layering as seen for $L=1$ (e.g., Figure 5a). It should be pointed out that $\left\langle V_{s}\right\rangle$ for $L=2$ is smaller than that for $L=1$ even though the degree of layering as measured by the ratio of $M_{v}$ to $M_{h}$ is less for $L=2$ than for $L=1$ (Table 2 and Figures $7 \mathrm{e}$ and $7 \mathrm{f}$ ). This is because an upwelling limb has developed under the downwelling limb and prevents the downwelling limb from penetration (Figures 10a and 11b). The unstable balance between the upwelling and downwelling limbs is maintained by the fixed vertical boundary where flow cannot move horizontally due to the reflecting boundary conditions; clearly, this is a model limitation and is probably irrelevant for Earth's mantle.

In order to further examine the effects of Clapeyron slope, three other cases for $L=3$ with different $\gamma(\gamma$ are $-0.12,-0.15$ and -0.21 for cases 23,24 , and 25 , respectively; see Table 2) were computed. Together with cases 17 and 21 in which $\gamma$ are -0.17 and -0.19 , respectively, we see that a steeper $\gamma$ results in a more sluggish convection, evident through a smaller $\left\langle M_{v}\right\rangle,\left\langle V_{s}\right\rangle$, and $\left\langle U_{p}\right\rangle$ (Table 2 and Figure 12a), and a larger degree of layered convection, evident through a smaller ratio of $\left\langle M_{y}\right\rangle$ to $\left\langle M_{h}\right\rangle$ and $R_{s}$ (Table 2 and Figure 12b). For $\gamma=$ -0.12 (case 23), the ratio of $\left\langle M_{v}\right\rangle$ to $\left\langle M_{h}\right\rangle$ and $R_{s}$ (Table 2 

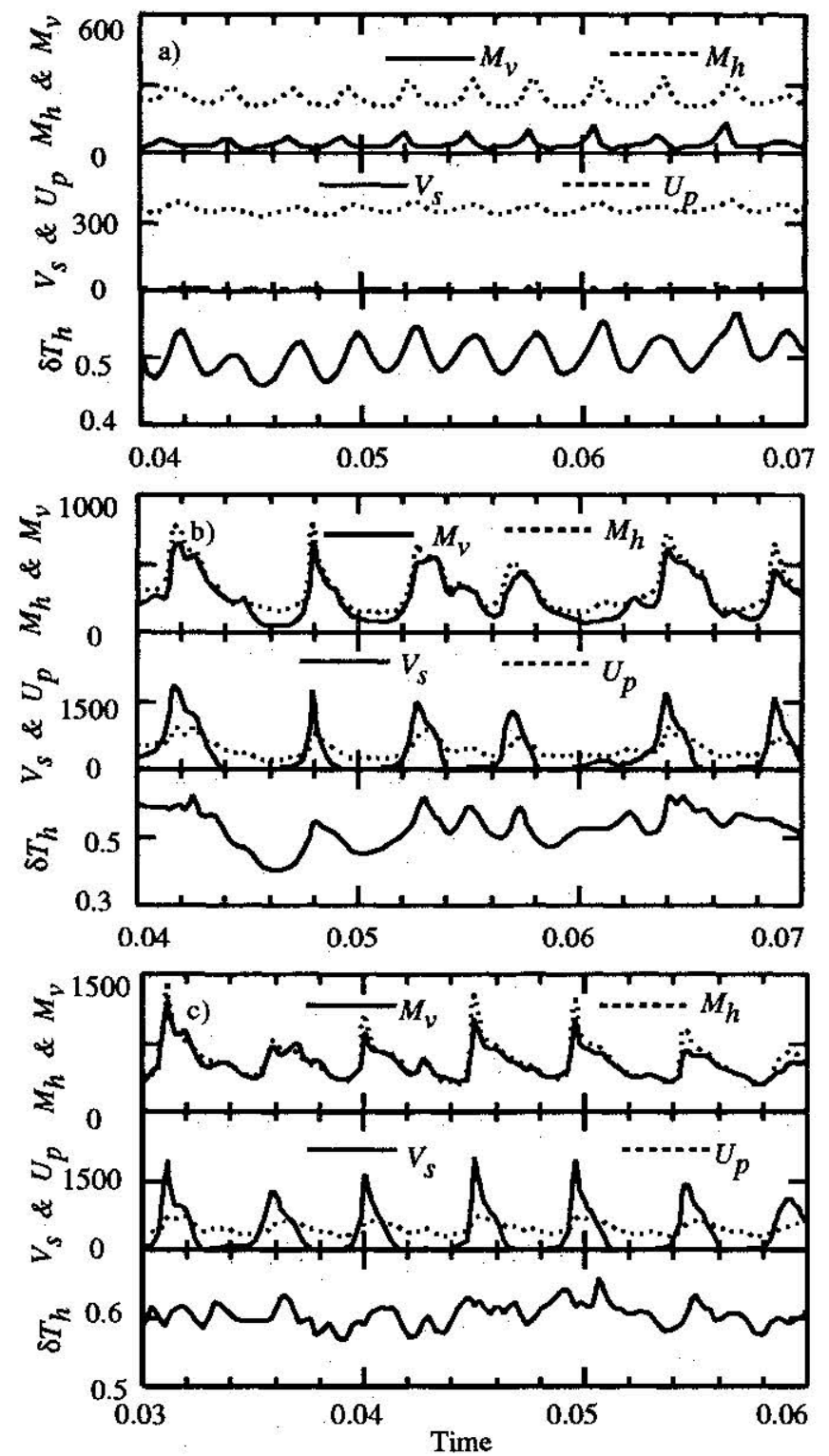

Figure 10. Time history of $M_{v}, M_{h}, V_{s}, U_{p}$, and $\delta T_{h}$ for cases with an increased strength of endothermic phase change and a plate of different length: (a) case 20 with $L=2$, (b) case 21 with $L=3$, and (c) case 22 with $L=5$.

and Figure 12b) indicate that the phase change is too weak to affect mass exchange across the phase change; the standard deviations of $M_{v}, V_{s}$, and $U_{p}$ are also relatively small compared with their time averages (Table 2 and Figure 12b), indicating a weak episodicity in thermal convection. For $\gamma=$ -0.21 (case 25), the ratio of $\left\langle M_{v}\right\rangle$ to $\left\langle M_{h}\right\rangle$ drops to 0.36 from 0.81 for the case with $\gamma=-0.19$ (case 21) (Figure 12b and Table 2), indicating significant layered convection. This ratio of $\left\langle M_{v}\right\rangle$ to $\left\langle M_{h}\right\rangle$ for $\gamma=-0.21$ is only slightly larger than the 0.33 for the isoviscous case with $\gamma=-0.17$ (case 10 in Table 2), indicating an important effect of $\gamma$ on the style of convection. $V_{s}$ and $R_{s}$ become negligibly small for $\gamma=$ -0.21 (Figure 13a for time history, Figure 12b and Table 2). The insignificant slab penetration is due to the formation of an upwelling limb in the lower layer beneath the downwelling within the upper layer (Figure $11 \mathrm{c}$ for representative temperature and stream functions), similar to that observed for the case with $\gamma=-0.19$ and $L=2$ (Table 2, Figures 10a and 11b). Interestingly, for $\gamma=-0.21$, the episodicity in thermal convection is also relatively weak, compared with those for small $\gamma$, except for $\gamma=-0.12$ (Figures $8 c, 10 b$, and 13a for cases with $\gamma=-0.17,-0.19$, and -0.21 , respectively), which is also evident through the ratios of standard deviations of $M_{v}, V_{s}$ and $U_{p}$ to their time averages (Figure 12b and Table 2 ). This suggests that a strong episodic convection induced by the phase change only occurs within a certain range of Clapeyron slopes.

The effects of plates when an additional exothermic phase change at $410 \mathrm{~km}$ depth is present are investigated with four cases of different plate length (cases 26,27,28, and 29 for $L=$ $1,2,3$, and 5, respectively; see Table 2 ). In these four cases, the exothermic phase changes have the same strength as the endothermic phase change $\left(R_{b}^{1}=R_{b}^{2}=2 \times 10^{6}, \gamma_{1}=0.17\right.$, and $\left.\gamma_{2}=-0.17\right)$. An exothermic phase change tends to counteract the effect of an endothermic phase change and enhances thermal convection [Schubert et al., 1975; Christensen and Yuen, 1985].

The results from these internally beated cases with two phase changes are similar to the results when only one endothermic phase change is present. A substantial increase in
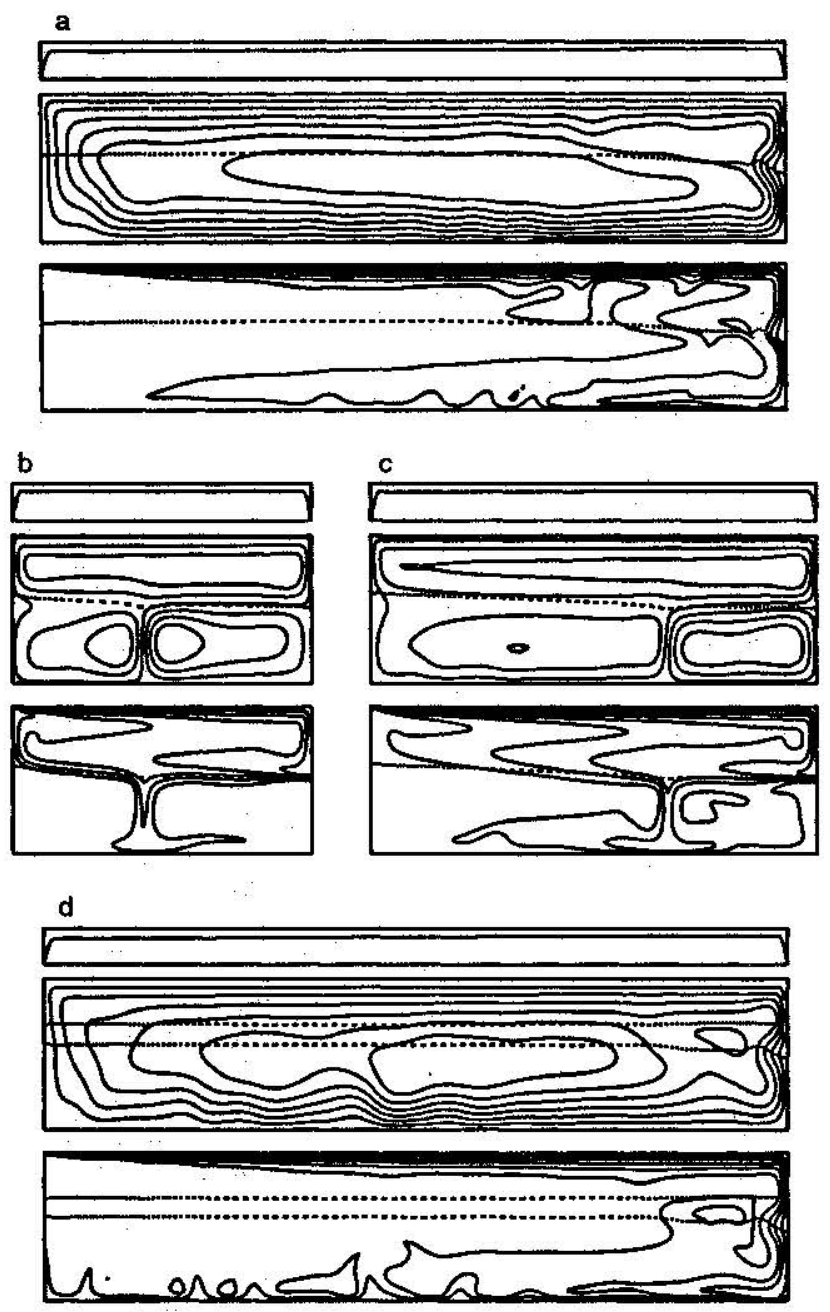

Figure 11. Representative temperature and stream functions for cases (a) 22 , (b) 20 , (c) 25 , and (d) 29 , respectively. An exthermic phase change is included in case 29 , and the exthermic phase change boundary is indicated by the upper dashed line (Figure 11d). For other details, see Figure 4. 

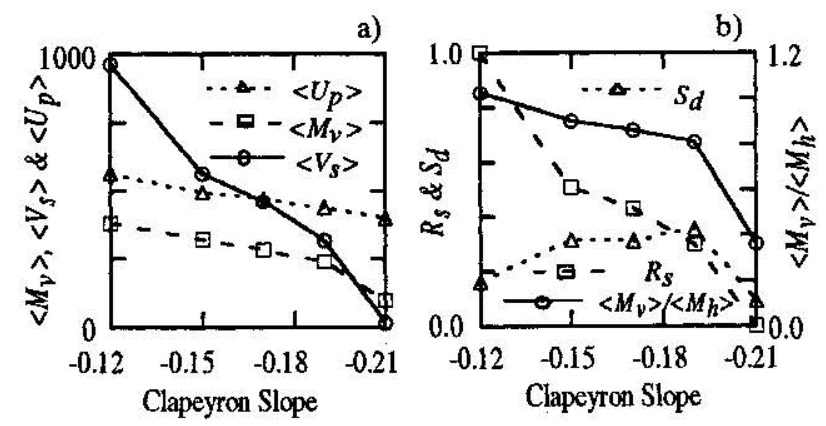

Figure 12. (a) $\left\langle M_{v}\right\rangle,\left\langle V_{s}\right\rangle,\left\langle U_{p}\right\rangle$, (b) the ratio of $\left\langle M_{v}\right\rangle$ to $\left\langle M_{h}\right\rangle, R_{s}$, and the ratio of the standard deviations of $U_{p}$ to $\left\langle U_{p}\right\rangle$ (i.e., $S_{d}$ ) versus Clapeyron slope for cases $17,21,23$, 24 and 25.

the mass exchange and slab penetration across the endothermic phase boundary, as plate length $L$ increases, is observed ( $R_{s},\left\langle M_{v}\right\rangle$, and $\left\langle V_{s}\right\rangle$ in Table 2). As $L$ increases, the ratio of $\left\langle M_{v}\right\rangle$ to $\left\langle M_{h}\right\rangle$ increases (Table 2), indicating a diminished effect of the endothermic phase change on mass exchange across the phase change for a larger $L$. Plate-scale flow structures always predominate (Figure 11d for case 29). Inclusion of the exothermic phase change results in more vigorous convection and more efficient heat transfer, compared to those cases with only an endothermic phase change (e.g., cases 15-18), evident from the increases in $\left\langle M_{v}\right\rangle,\left\langle V_{s}\right\rangle,\left\langle U_{p}\right\rangle,\left\langle\delta T_{h}\right\rangle, R_{s}$ and $F$ (Table 2).

It is important to realize that the material property method always yields a "plate-like" surface velocity with a plateness close to 0.9 for models with different plate lengths, different phase change parameters, and different heating modes (Table 2 and Figures 5, 9, and 11). The continuous cooling of the top thermal boundary layer is well simulated (Figures 5, 9, and 11), although minor boundary layer instabilities start to develop close to the major downwellings for $L=5$ (e.g., Figures $5 \mathrm{c}$ and $11 \mathrm{a}$ ). The plate velocity $U_{p}$ and the mass flux $M_{v}$ are often both correlated with $\delta T_{h}$ (see all the history plots). $\left\langle U_{p}\right\rangle$ increases with plate length $L$ (Table 2 ), as expected from the increase of $\left\langle\delta T_{h}\right\rangle$ with $L$ (Table 2). Plate motion is controlled by plate-scale structures which are generated due to the presence of plates. This is similar to the results reported by Zhong and Gumis [1993].

\section{Effects of Stiff Slabs}

We have shown that stiff plates have important effects on phase change dynamics. Stiff plates result from temperaturedependent rheology of the Earth's mantle, and the same rheology will make subducted slabs stiff. Because a stiff subducted slab will tend to retain its shape, slab strength may have important effects on phase change dynamics, including slab penetration [Christensen and Yuen, 1984]. In this section, we shall use temperature-dependent rheology to investigate the effects of stiff slabs, which were not addressed in our previous material property plate models.

For proper comparisons between different cases, it is essential to have the same thermal Rayleigh number, since effects of phase changes on mass exchange across endothermic phase change depend on the Rayleigh number [Christensen and Yuen, 1985; Zhao et al., 1992]. $R_{a}$ was $10^{6}$ for previous cases with dynamic plates; for temperaturedependent viscosity cases, $R_{a}$ defined with the viscosity at the bottom boundary temperature is chosen such that the $\boldsymbol{R}_{a}$ defined with the averaged viscosity over the domain excluding the region for the high-viscosity lids is approximately $10^{6}$.

While temperature-dependent viscosity makes both plates and subducted slabs stiff, the resulting surface velocity does not resemble "plate-like" velocity [e.g., Gurnis, 1989]; the magnitude of surface velocity is negligibly small, and there is significant interior strain. This is illustrated by a case with temperature-dependent viscosity, TDV1 (Figure 2), and a free slip boundary condition (case 30 in Table 2; see Figure $4 \mathrm{c}$ for representative temperature and flow fields and surface velocity). The upper layer due to its relatively low temperature is much less mobile than the lower layer (Figure $4 \mathrm{c}$ ), and the average surface velocity is 74 (Table 2), significantly smaller than the bottom average velocity of 300 and also much smaller than the average surface velocity for the isoviscous flow model (case 10 in Table 2) which only differs from this temperature-dependent viscosity case (case 30 ) in rheology. Moreover, there is significant interior strain in surface velocity (Figure 4c). Compared with the isoviscous case (case 10 ), the degree of layering as measured by the ratio of $\left\langle M_{v}\right\rangle$ to $\left\langle M_{h}\right\rangle$ only changes slightly (Table 2); the immobile top boundary results in a substantial decrease in surface heat flux $F$ (Table 2). The significantly high internal heating rate for case 30 is due to the relatively uniform temperature in the lower layer (Figure $4 \mathrm{c}$ ), which stifles the flow of heat into the bottom boundary.

In order to obtain a realistic plate in temperature-dependent viscosity models, a plate generation method is necessary. Following Davies [1988a], a constant velocity is imposed on
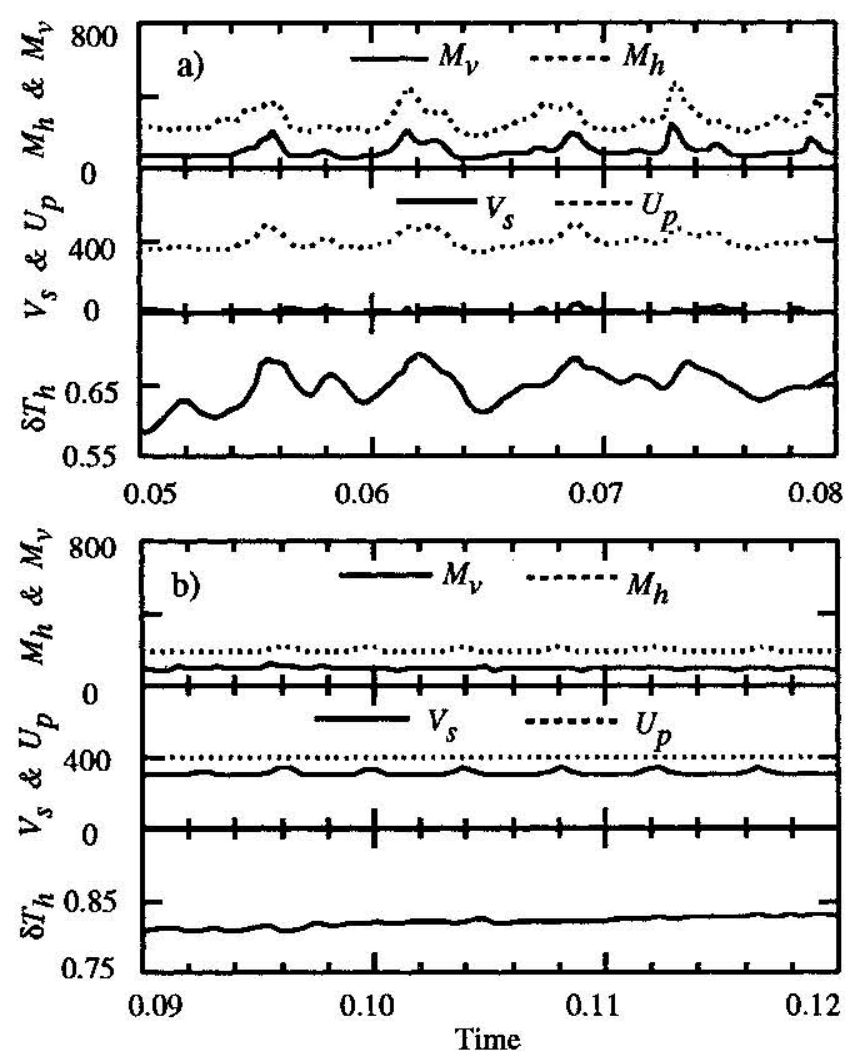

Figure 13. Time history of $M_{v}, M_{h}, V_{s}, U_{p}$, and $\delta T_{h}$ for cases with $\gamma=-0.21$ but with different rheology: (a) case 25 with the material property method and (b) case 35 with a temperature-dependent viscosity, TDV1. 
the surface as a boundary condition. The surface velocity has to be chosen properly such that on average over a long period of time no significant amount of extra kinetic energy is introduced into the system. Before introducing the imposed surface velocity plate into temperature-dependent viscosity models, we will first demonstrate that if the averaged plate velocity from a material property model is taken as the imposed velocity for a model with a same Rayleigh number and Clapeyron slope, the imposing surface velocity does not significantly alter the dynamics of the system.

Two cases (cases 31 and 32 in Table 2) with imposed constant surface velocities on high-viscosity lids without weak margins are computed. The imposed surface velocities for cases 31 and 32 are the averaged plate velocities for cases 7 and 17 (Table 2), respectively; the latter two cases with dynamic plates differ from the former two only in having two weak margins and a free-slip top surface. Both the timeaveraged quantities including $\left\langle M_{v}\right\rangle,\left\langle M_{h}\right\rangle,\left\langle V_{s}\right\rangle, R_{s}, F, \xi$, and $T_{m}$ and their time -dependencies for these two imposed surface velocity cases (cases 31 and 32 ) are nearly identical to those for models with dynamic plates (cases 7 and 17) (Table 2 and Figures $8 \mathrm{c}$ and $14 \mathrm{a}$ for time dependence for cases 17 and 32 , respectively).

Given the above justifications of using imposed surface velocity, we then introduce temperature-dependent rheology along with the imposed surface velocity into three cases (cases 33-35). Cases 33, 34, and 35 with temperature-dependent rheology differ from previous cases 7,17 , and 25 (Table 2), respectively, in only the rheology and surface boundary conditions; the imposed surface velocities for these temperature-dependent viscosity cases are the averaged plate velocities for previous cases with dynamic plates (Table 2). The temperature dependence of viscosity for these three cases (cases 33-35) is the TDV1 (Figure 2). $M_{v}$ and $V_{s}$ for the temperature-dependent viscosity case with no phase change (case 33) decrease, but heat flux $F$ increases, compared to those for the case with material property plates (case 7) (Table 2 ). The higher viscosity of slabs tends to decrease $V_{s}$ and makes $V_{s}$ closer to the plate velocity (Table 2).

For the cases with the phase change, temperature-dependent viscosity results in significant changes in slab penetration and the episodicity of convection, compared with cases with only stiff plates (see time dependence, Figures $8 \mathrm{c}$ and $14 \mathrm{~b}$ for cases 17 and 34 , respectively, with $\gamma=-0.17$; Figures 13a and $13 \mathrm{~b}$ for cases 25 and 35 , respectively, with $\gamma=-0.21$ ). For case $34, R_{s}=1.0 ; V_{s}$ is always close to $U_{p}$ with insignificant episodicity (Table 2 and Figure 14b), indicating that the downwelling continuously penetrates the phase boundary (Figure 15a for representative temperature and flow fields). This is significantly different from case 17 with only stiff plates, in which case, slab penetration is frequently stopped by the phase change with a strong episodicity (Figures $8 \mathrm{c}$ and 9). More dramatic changes resulting from stiff slabs in slab penetration occur when $\gamma=-0.21$ (cases 25 and 35). In case 25 with no stiff slab, slabs cannot penetrate through the phase change (Figure 11c for temperature and flow fields and Figure 13a for time dependence), but slabs penetrate steadily through the phase change when slabs are made stiff through temperature-dependent viscosity in case 35 (Figure $13 \mathrm{~b}$ for time dependence and Figure $15 \mathrm{~b}$ for representative temperature and flow fields). $R_{S}$ is 0.26 for case 35 (Table 2). This is due to the definition of $R_{s}$, in which the occurrence of penetration is when $V_{s}$ is larger than 0.8 of $U_{p}$; indeed, $V_{s}$ is always about
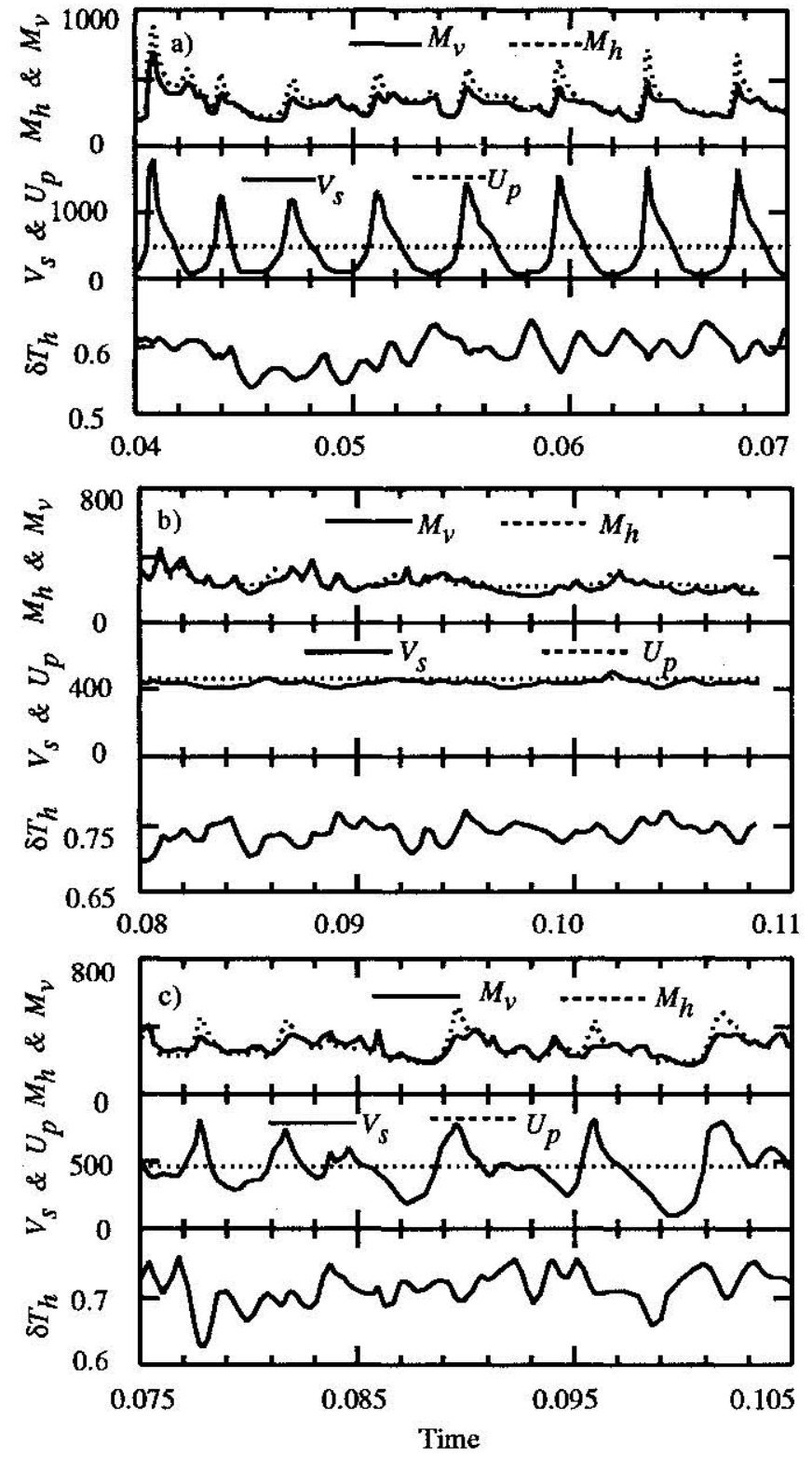

Figure 14. Time history of $M_{v}, M_{h}, V_{s}, U_{p}$, and $\delta T_{h}$ for cases with $\gamma=-0.17$ but with different rheology: (a) case 32 with a high viscosity plate of imposed surface velocity, (b) case 34 with TDV1, and (c) case 36 with TDV2.

0.75 of $U_{p}$ (Figure 13b). The episodicity in thermal convection is substantially diminished for this temperaturedependent viscosity case (case 35 ), compared to the case with no stiff slabs (case 25). Interestingly, the temperaturedependent rheology only slightly reduces the degree of layering, as indicated by the ratios of $\left\langle M_{v}\right\rangle$ to $\left\langle M_{h}\right\rangle$ (Table 2 ). For case 35 , even though slabs steadily penetrate through the phase change due to temperature-dependent viscosity, convection is still significantly layered, evident through the ratio of $\left\langle M_{v}\right\rangle$ to $\left\langle M_{h}\right\rangle$, which is 0.47 (Table 2), the time dependence of $M_{v}$ and $M_{h}$ (Figure 13b), and the representative flow field (Figure 15b).

Comparison between the temperature-dependent viscosity cases with a plate (case 34) and that with no plate (case 30) reveals the effects of a plate. Case 34 differs from case 30 only in having a mobile plate by imposing surface velocity. The 
a
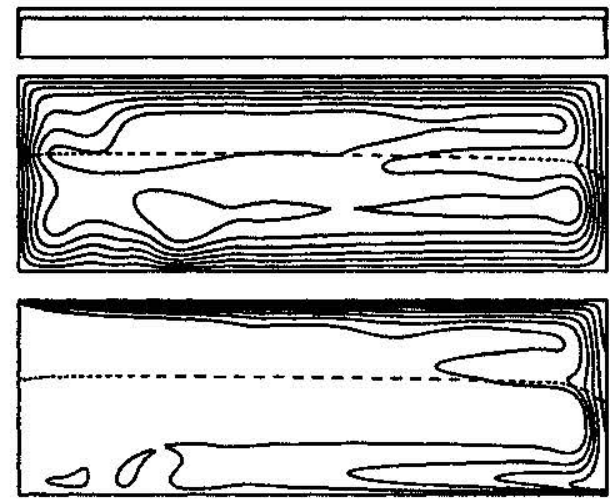

b
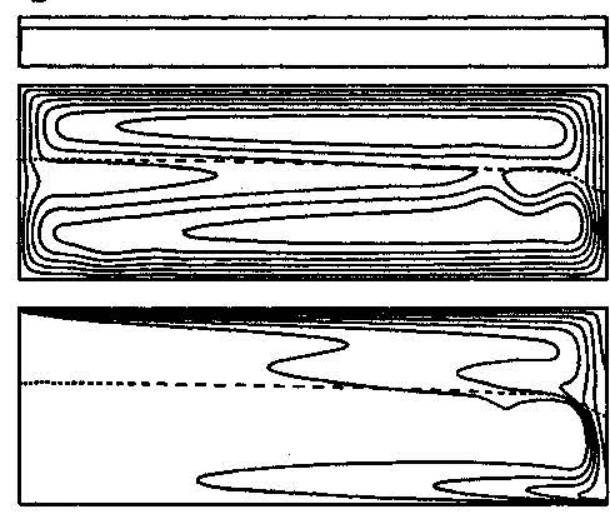

C
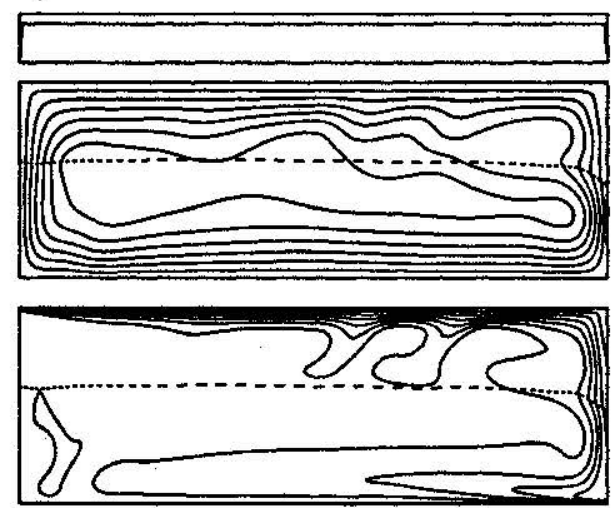

Figure 15. Representative temperature and stream functions for temperature-dependent viscosity cases: (a) case 34 , (b) case 35 , and (c) case 36, respectively. For other details, sec Figure 4.

ratio of $\left\langle M_{v}\right\rangle$ to $\left\langle M_{h}\right\rangle$ is 0.93 for case 34 but is only 0.28 for case 30 (Table 2), indicating that the presence of plates significantly reduces the degree of layering, which is also evident in the flow fields (Figures $4 c$ and 15a). Together with the observation that rheology can significantly change slab penetration but has little effects on the degree of layering for a given plate length (e.g., cases 25 and 36 for $\gamma=-0.21$ in Table 2), this again suggests that global features including mass flux and the degree of layering are controlled by largescale structures organized by plates, while slab penetration is more related to local features.

Temperature-dependent viscosity gives rise to high strength slabs; slabs with high strength tend to retain their integrity and thus enhance slab penetration. Slab penetration depends on the strength of slabs. The effect of a different temperature- dependence of viscosity is examined in case 36 which only differ from case 34 by using a different temperature dependence of viscosity, TDV2 (Figure 2). Since TDV2 gives rise to slabs which are weaker than those from TDV1 (case 34) but stronger than those of constant background viscosity (case 17), the results from the case with TDV2 (case 36) are between those from the case with TDV1 (case 34) and those from the case with no stiff slabs (case 17). Thermal convection becomes more episodic, compared to the case with TDV1 (case 34); slab penetration velocity $V_{s}$ significantly fluctuates, although the fluctuation is smaller than that for the case with no stiff slabs (case 17) (Figure $14 \mathrm{c}$ is time dependence for case 36 with TDV2). It should be pointed out that significant thermal boundary layer instabilities occur for the case with TDV2 (case 36) (Figure $15 \mathrm{c}$ for representative temperature and flow fields), which may result in leakage in negative buoyancy within plates and thus weakens slab penetration.

\section{Conclusions}

Our thermal convection models with multiple phase changes and realistic plates and slabs have consistently shown for different Clapeyron slopes and different heating modes that plates and stiff slabs have a profound effect on phase change dynamics. As plate length increases, slab penetration occurs more frequently; convection becomes less layered; mass flux across endothermic phase change increases. A stiff slab due to temperature-dependent rheology enhances slab penetration but has little effect on total mass flux and degree of layering. These results are of particular importance to the dynamics of western Pacific subduction zones where plate sizes are large.

Plates always generate predominant plate-scale thermal and flow structures, independent of plate length. Plate-scale structures have important controls over the style of convection. As plates become larger, the resulting larger scale structures are less influenced by endothermic phase changes, thus reducing the degree of layering. Our models show that complete layered convection occurring for a plate length equal to the thickness of the box becomes insignificantly layered as the plate length increases to 3 or 5 times larger and that the time ratio of slab penetration can increase from zero for cases with small plates to more than 0.5 for cases with large plates.

We suggest that large-scale features including degree of layering, plate velocity, and mass flux are controlled by largescale structures, while slab penetration may be related more to small-scale features. Therefore, whether or not subducted slabs penetrate the phase change may not necessarily indicate that convection is layered or unlayered. Unlayered convection can coexist with no slab penetration (e.g., Figure $6 \mathrm{c}$ for case 14) and steady slab penetration can coexist with significantly layered convection (e.g., Figures $13 \mathrm{~b}$ and $15 \mathrm{~b}$ for case 35).

The episodicity of convection induced by the endothermic phase changes strongly depends on plate length, rheology, and Clapeyron slope. As long as plate size increases to a certain length, further increases in plate length would weaken the episodicity of convection (e.g. Figures 6,8 and 10). When slabs become stiff with temperature-dependent rheology, episodicity in convection also weakens substantially (e.g., Figure 13a versus Figure 13b and Figure 8c versus Figure 14b). Only for a certain range of Clapeyron slope, can the phase 
change induce a strong episodic thermal convection; any endothermic phase change with Clapeyron slope larger or smaller than this range tends to give a more steady convection (Figure 12b).

The proper simulations of plates and subducted slabs are essential for mantle convection models. The material property method, because it effectively takes into account both temperature- and stress- dependent rheology, provides a very successful tool to simulate plates even for a plate as long as 5 times the thickness of the fluid layer. Imposed surface velocity together with temperature-dependent viscosity can simulate both plates and stiff slabs, although it is computationally costly.

Acknowledgment. We appreciate the discussions with S. Weinstein and reviews by $S$. King, $G$. Davies, and an anonymous reviewer. Many of the computations were carried at the Pittsburgh Supercomputer Center. Funded by the David and Lucile Packard Foundation and NSF grant EAR-8957164.

\section{References}

Brooks, A. N., A Petrov-Galerkin finite element formulation for convection dominated flows, Ph.D. thesis, Calif. Inst. of Technol., Pasadena, 1981.

Cazenave, A., and B. Lago, Long wavelength topography, seafloor subsidence and flattening, Geophys. Res. Lett., 18, 1257-1260, 1991.

Christensen, U. R., and D. A. Yuen, Layered convection induced by phase transitions, J. Geophys. Res., 90, 10,291-10,300, 1985.

Christensen, U. R., and D. A. Yuen, The interaction of a subducting lithospheric slab with a chemical or phase boundary, J. Geophys. Res., 89, 4389-4402, 1984.

Davies, G. F., Role of the lithosphere in mantle convection, J. Geophys. Res., 93, 10,451-10,466, 1988a.

Davies, G. F., Ocean bathymetry and mantle convection, 1, Large-scale flow and hotspots, J. Geophys. Res., 93, 10,467-10,480, 1988b.

Davies, G. F., Ocean bathymetry and mantle convection, 2 , Small-scale flow, J. Geophys. Res., 93, 10,481-10,488, 1988c.

Davies, G. F. and F., Pribac, Mesozoic seafloor subsidence and the Darwin Rise, past and present, in The Mesozoic Pacific: Geology, Tectonics, and Volcanism, Geophys. Monogr. Ser., vol. 77, edited by M. Pringle, W. Sager, W. Sliter and S. Stein, pp. 39-52, AGU, Washington, D. C., 1993.

Gable, C. W., R. J. O'Connell, and B. J. Travis, Convection in three dimensions with surface plates: Generation of toroidal flow, $J$. Geophys. Res., 96, 8391-8405, 1991.

Gurnis, M., A reassessment of the heat transport by variable viscosity convection with plates and lids, Geophys. Res. Lett., 16, 179-182, 1989.

Gurnis, M., and B. H. Hager, Controls on the structure of subducted slabs, Nature, 335, 317-321, 1988.

Heestand, R. L., and S. T. Crough, The effect of hotspots on the oceanic age-depth relation, J. Geophys. Res., 86, 6107-6114, 1981.

Honda, S., S. Balachandar, D. A. Yuen, and D. Reuteler, Threedimensional instabilities of mantle convection with multiple phase transitions, Science, 259, 1308-1311, 1993.
Hughes, T. J. R., The Finite Element Method, 631 pp., Prentice-Hall, Englewood Cliffs, N.J., 1987.

King, S. D., C. W. Gable, and S. A. Weinstein, Models of convection driven tectonic plates: A comparison of methods and results, Geophys. J. Int., 109, 481-487, 1992.

King, S. D., and B. H. Hager, The relationship between plate velocity and trench viscosity in newtonian and power-law subduction calculations, Geophys. Res. Lett., 17, 2409-2412, 1990.

King, S. D., Raefsky, A. and Hager, B. H., ConMan: Vectorizing a finite element code for incompressible two-dimensional convection in the Earth's mantle, Phys. Earth Planet. Inter., 59, 195-207, 1990.

Machetel, P., and P. Weber, Intermittent layered convection in a model with an endothermic phase change at $670 \mathrm{~km}$, Nature, 350, 55-57, 1991.

McKenzie, D. P., J. M. Roberts, and N. O. Weiss, Convection in the Earth's mantle: Towards a numerical simulation, J. Fluid Mech., 62, 465-538, 1974.

Parsons, B., and J. G. Sclater, An analysis of the variation of ocean floor bathymatry and heat flow with age, J. Geophys. Res., 82, 803-827, 1977.

Peltier, W. R., and L. P. Solheim, Mantle phase transitions and layered chaotic convection, Geophys. Res. Lett., 19, 321-324, 1992.

Richter, F. M., Finite amplitude convection through a phase boundary, Geophys. J. R. Astron. Soc., 35, 265-276, 1973.

Schroeder, W., The empirical age-depth relation and depth anomalies in the Pacific Ocean basin, J. Geophys. Res., 89, 9873-9884, 1984.

Schubert, G., D. A. Yuen, and D. L. Turcotte, Role of phase transitions in a dynamic mantle, Geophys. J. R. astr. Soc., 42, 705-735, 1975.

Su, W.-J., and A. M. Dziewonski, On the scale of mantle heterogeneity, Phys. Earth Planet. Inter., 74, 29-54, 1992.

Tackley, P. J., D. J. Stevenson, G. A. Glatzmeir, and G. Schubert, Effects of an endothermic phase transition at $670 \mathrm{~km}$ depth on spherical mantle convection, Nature, 361, 137-160, 1993.

Weinstein, S. A., Catastrophic overturn of the Earth's mantle driven by multiple phase changes and internal heat generation, Geophys. Res. Lett., 20, 101-104, 1993.

Weinstein, S. A., and P. L. Olson, Thermal convection with nonNewtonian plates, Geophys. J. Int., 111, 515-530, 1992.

Woodward, R. L., and G. Masters, Global upper mantle structure from ScS-S differential travel times, J. Geophys. Res., 96, 6351-6377, 1991.

Zhang, Y.S., and T. Tanimoto, Global Love wave phase velovity variation and its significance to plate tectonics, Phys. Earth Planet. Inter, 66, 160-202, 1991.

Zhao, W., D. A. Yuen, and S. Honda, Multiple phase transitions and the style of mantle convection, Phys. Earth Planet. Inter., 72, 185-210, 1992.

Zhong, S., and M. Gurnis, Dynamic feedback between an nonsubducting raft and thermal convection, J. Geophys. Res., 98, 12,219-12,232, 1993.

M. Gurnis, Seismological Laboratory, California Institute of Technology, Pasadena, CA 91125.

S. Zhong, Department of Geological Sciences, University of Michigan, Ann Arbor, MI 48109-1063.

(Received September 1, 1993; revised January 31, 1994; accepted February 18, 1994.) 